\title{
Imaging FTS: A Different Approach to Integral Field Spectroscopy
}

\author{
Laurent Drissen, ${ }^{1}$ Laurie Rousseau-Nepton, ${ }^{1}$ Sébastien Lavoie, ${ }^{1}$ Carmelle Robert, ${ }^{1}$ \\ Thomas Martin, ${ }^{1}$ Pierre Martin, ${ }^{2}$ Julie Mandar, ${ }^{3}$ and Frédéric Grandmont ${ }^{3}$ \\ ${ }^{1}$ Département de Physique, de Génie Physique et d'Optique, Université Laval and Centre de Recherche en Astrophysique du \\ Québec (CRAQ), 1045 Avenue de la Médecine, Québec, QC, Canada G1V 0A6 \\ ${ }^{2}$ Department of Physics and Astronomy, University of Hawaii at Hilo, 200 W. Kawili Street, Hilo, HI 96720-4091, USA \\ ${ }^{3}$ ABB Analytical, 585 Boulevard Charest Est, Suite 300, Québec, QC, Canada G1K $9 H 4$
}

Correspondence should be addressed to Laurent Drissen; ldrissen@phy.ulaval.ca

Received 15 November 2013; Accepted 24 February 2014; Published 22 April 2014

Academic Editor: Polychronis Papaderos

Copyright $\odot 2014$ Laurent Drissen et al. This is an open access article distributed under the Creative Commons Attribution License, which permits unrestricted use, distribution, and reproduction in any medium, provided the original work is properly cited.

\begin{abstract}
Imaging Fourier transform spectroscopy (iFTS) is a promising, although technically very challenging, option for wide-field hyperspectral imagery. We present in this paper an introduction to the iFTS concept and its advantages and drawbacks, as well as examples of data obtained with a prototype iFTS, SpIOMM, attached to the $1.6 \mathrm{~m}$ telescope of the Observatoire du Mont-Mégantic: emission line ratios in the spiral galaxy NGC 628 and absorption line indices in the giant elliptical M87. We conclude by introducing SpIOMM's successor, SITELLE, which will be installed at the Canada-France-Hawaii Telescope in 2014.
\end{abstract}

\section{Introduction}

The vast majority of astronomical imaging spectrometers (or integral field spectrographs) are based on dispersive approaches. We present in this paper the most recent technical developments and some scientific results based on another approach, imaging Fourier transform spectroscopy (iFTS), which has been given a strong boost during the past decade, mostly because of enormous improvements in digital imaging capabilities, computer power, and servo control systems. A large number of research programs will certainly benefit from an instrument capable of simultaneously obtaining spatially resolved spectra on extended areas ( 10 arcminutes) with a $100 \%$ filling factor, seeing-limited spatial resolution, and a flexible spectral resolution up to $R \sim 10^{4}$, and iFTS is very promising in that regard. Single-pixel FTS has been in regular use in commercial applications, remote sensing of the Earth's atmosphere, and astronomy, mostly in the infrared and sub-mm domain. However, by using appropriate optical configurations, fast readout CCD detectors, and especially improved metrology and servo systems, it is possible to transform the traditional one-pixel infrared FTS into a truly integral field spectrometer for the visible range.

The most noteworthy scientific result from the use of the FTS approach in astronomy is the accurate measurement of the cosmic microwave background radiation spectral distribution, by the FIRAS instrument on the COBE satellite [1] and the COBRA rocket experiment [2]. On a completely different field, high resolution spectra of late-type stars were obtained on a regular basis with the FTS at Kitt Peak's Mayall telescope [3,4]. A high-resolution FTS was one of the first instruments to be attached to the Canada-FranceHawaii Telescope and was widely used on a large variety of planetary and stellar programs $[5,6]$. Combined with an imaging system in the early 1990s, it was renamed BEAR [7] and provided integral field spectra of a variety of objects such as planetary nebulae, massive star clusters, and starforming regions in a 24-arcsecond field of view [8]. More recent examples include SPIRE-FTS, one of three instruments to fly on ESA's Herschel Space Observatory [9], a far-infrared FTS on the Japanese satellite AKARI, a mid-IR FTS (CIRS) on the Cassini spacecraft, and, for the near IR, PFS on Mars 
Express with a copy on Venus Express. The development of imaging FTS in astronomy was given a strong incentive during the early definition phases of the NGST (now known as the James Webb Space Telescope): astronomers supported by the three participating space agencies (NASA, ESA, and the Canadian Space Agency) presented studies of imaging FTS at the NGST Instrumentation meeting in Hyannis in 1999 [10-12]. None of these concepts however was included in the final instrument suite of the telescope. Our involvement in the iFTS endeavour is a direct consequence of this meeting. More recently, Boulanger et al. [13] proposed the design of a $1.2 \mathrm{~m}$ space telescope, H2EX, equipped with a widefield imaging FTS specifically aimed at studying molecular hydrogen in the Universe. The advantages and disadvantages of the imaging FTS technique, as well as the relative merit of different approaches to 3D imagery, are discussed by Bennett [14]. An earlier version of the present paper, more complete in terms of technical explanations, is presented by Drissen et al. [15]. A recent review of the imaging FTS concept, with some historical perspective, a detailed comparison between the various imaging spectroscopy concepts and technical details not discussed in the present paper, is presented by Maillard et al. [16].

\section{The iFTS Concept}

An astronomical imaging Fourier transform spectrometer is basically a Michelson interferometer inserted into the collimated beam of an astronomical camera system, equipped with two detectors. The Michelson interferometer consists of a beamsplitter used to separate the incoming beam into two equal parts, two mirrors on which the halves of the original beam are reflected back, a moving mechanism to adjust the position and orientation of one of the mirrors (the other mirror is fixed), and a metrology system (IR laser and detector) to monitor the mirror alignment. All wavelengths from the field are simultaneously transmitted to either one or both of the interferometer outputs in which the array detectors sit. By moving one of its two mirrors, the interferometer thus configured therefore modulates the scene intensity between the two outputs instead of spectrally filtering it. This configuration results in a large light gathering power since no light is lost except through items common to any optical design: substrate transmission, coatings efficiency, and quantum efficiency of the detectors. All photons from the source can hence be recorded at each exposure provided that both complementary outputs of the interferometer are recorded. This requires a modification to the "standard" Michelson configuration in which half the light goes back to the source: the incoming light enters the interferometer at an angle allowing the two output beams to be physically separated. A CCD detector is then attached to each of the two output optics collecting the light from the interferometer (see Figure 6 in [15]).

While in most FTSs targeting very bright sources the interferometer's mirror is moved at a regular, servoed speed, the weak signal from astronomical sources requires a stepscan approach. The interferogram cube is obtained through the acquisition of a series of short exposure images with the two CCDs. At each step, one of the two mirrors in the interferometer is moved by a very short distance (between $175 \mathrm{~nm}$ and $\sim 5 \mu \mathrm{m}$, depending on the spectral resolution and waveband chosen). The signal at each pixel is modulated, as a function of the mirror position, by a pattern which depends on the spectral content of the source; each of the detectors'pixels is recording at each step a signal complementary to the corresponding pixel on the other detector. The vector composed of such a pixel recording is called an interferogram and is uniquely determined by the spectral content of the incoming light. The sum of the two images acquired at each step by the two detectors is then identical to a single image obtained with a "normal" camera. Spectral information for every pixel is recovered through a Discrete Fourier Transform (DFT or FFT) through the interferogram cube which can at any time during acquisition be turned into a spectral cube since each image contains information covering the whole waveband. The inclusion of additional exposures to an interferogram cube simply refines the meshing of the output spectra (spectral resolution). Thus, with an iFTS, spectral resolution is proportional to the total optical path difference (OPD) between the two arms of the interferometer scanned between the first and the last image of the data cube; this OPD needs to be properly sampled through a series of mirror displacementsat predetermined sequential interference positions. Once the data cube has been obtained and its individual images corrected for instrumental artifacts (bias, Flatfield, like for any imager), the Fourier transform of each pixel's interferogram produces a wavelength-calibrated data cube. A by-product of the spectral data cubes is therefore a deep panchromatic image (within the limits of the filter used-see below) of the targets. Figure 1 summarizes the data acquisition with an iFTS and Figure 2 shows a tangible example.

2.1. Technical Challenges. Like every imager, an iFTS must include high transmission optics producing high quality panchromatic images across the entire waveband covered by the instrument. But this does not translate alone into a good spectroscopic performance. In order to perform well on this aspect, a good modulation efficiency is also required. The performance of an FTS is thus characterized by its modulation efficiency (ME), that is, the capability of the interferometer to modulate the incident light:

$$
\mathrm{ME}=\frac{I \text { (modulated light })}{I \text { (incoming light })} \text {. }
$$

The modulation efficiency can be viewed as an analog to the grating efficiency in dispersive spectrographs. In the worst case scenario, where the modulation efficiency is zero, the light from the source is recorded on the detector but the interferogram is a straight line and no spectral information can be extracted from it.

This efficiency depends on a multitude of factors, the most technically challenging being the following.

(1) The surface quality of the optical components in the interferometer (mirrors and beamsplitter): at a given 


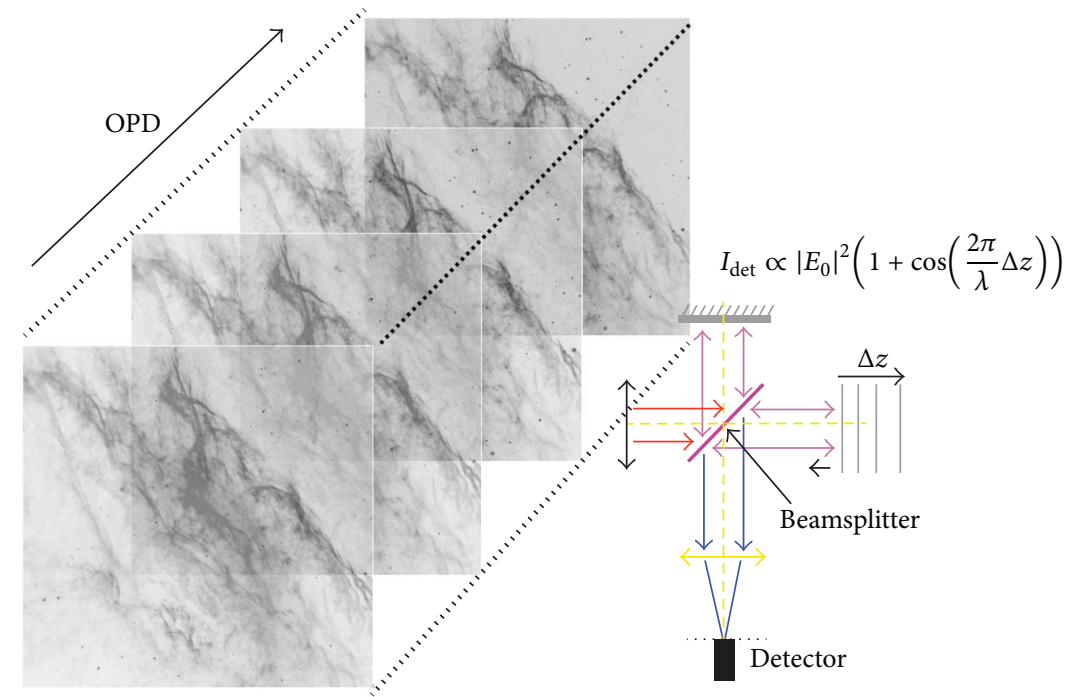

(a)

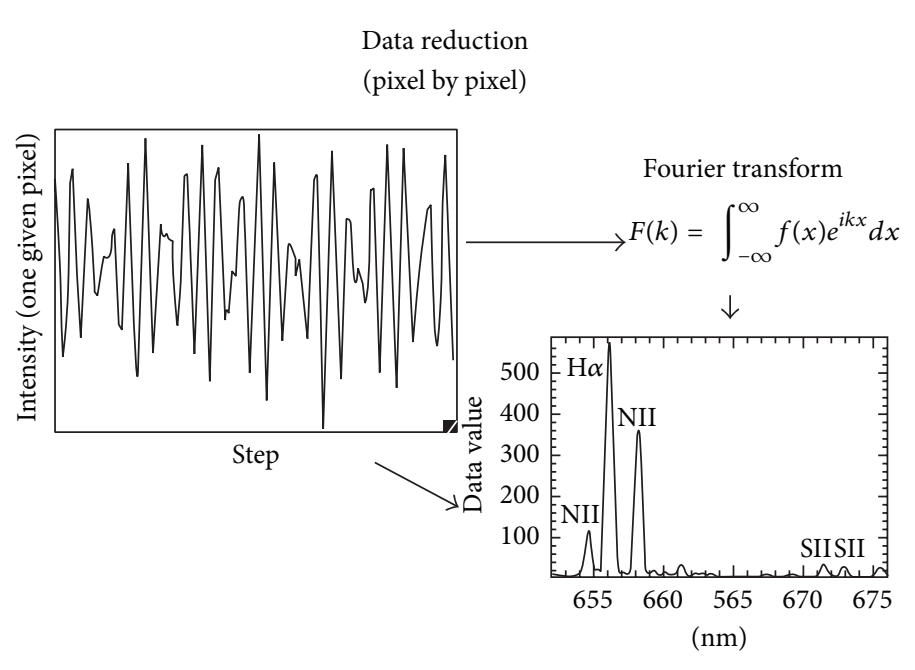

(b)

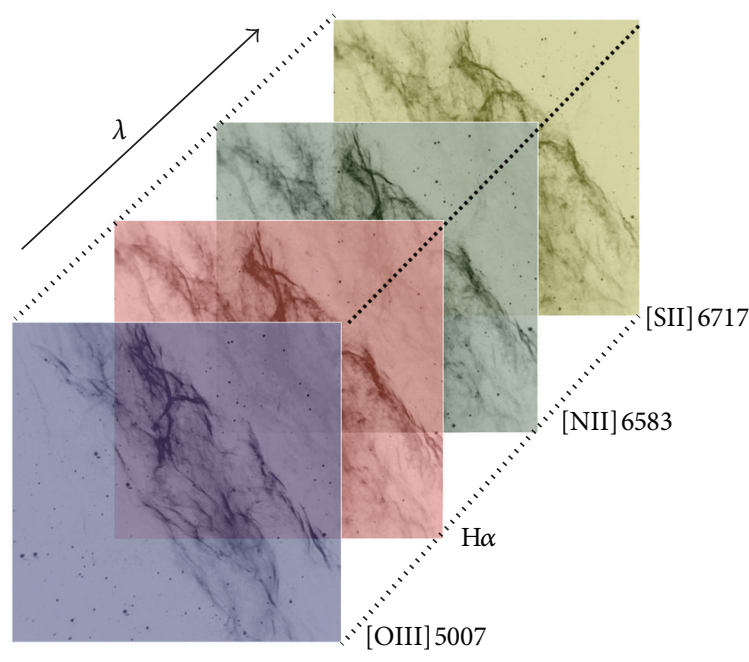

(c)

FIGURE 1: Data acquisition with an imaging FTS. (a) By scanning the Optical Path Difference (OPD) of the interferometer and taking images at every step, one gets a datacube composed of one interferogram for every pixel. (b) For a given pixel, the recorded intensity varies as a function of the OPD with a pattern that depends on the spectral content of the source; for example, a monochromatic laser beam would produce a sinusoidal pattern. A Fourier transform of the signal produces a spectrum for every pixel in the image. (c) After Fourier transforming every interferogram, one gets a spectral datacube from which monochromatic images corresponding to the emission lines of interest are extracted.

wavelength, ME is lowered by a decreased surface quality; it is therefore more and more difficult to obtain a good ME as we move from the infrared to the ultraviolet (most FTSs available today indeed work at infrared and sub-mm wavelengths); the number of reflections within the interferometer plays a major role in the global ME. Mirrors with a surface quality of $\lambda / 20$ (peak-to-valley) are commercially available for a reasonable price, but large $\lambda / 30$ mirrors must be custom made and are therefore much more expensive. In the case of a flat mirror design such as SpIOMM (see below), the ME at $350 \mathrm{~nm}$ doubles (from 35\% to $70 \%)$ as the mirror and beamsplitter surface quality improves from $\lambda / 20$ to $\lambda / 30$; at $800 \mathrm{~nm}$, the improvement is not as large (from $85 \%$ to $92 \%$ ). Moreover, even if the mirror substrate is of high enough quality, any error in the coating deposit or any tension caused by the mechanical parts used to maintain the mirror within the interferometer can ruin the initial surface figure and dramatically reduce the modulation efficiency, especially in the blue part of the spectral range.

(2) The mirror alignment and the stability of the OPD during an exposure: both crucially depend on the quality of the metrology and the servo system, which represents the highest technical challenge a visibleband iFTS faces. In order for the beams from the two 

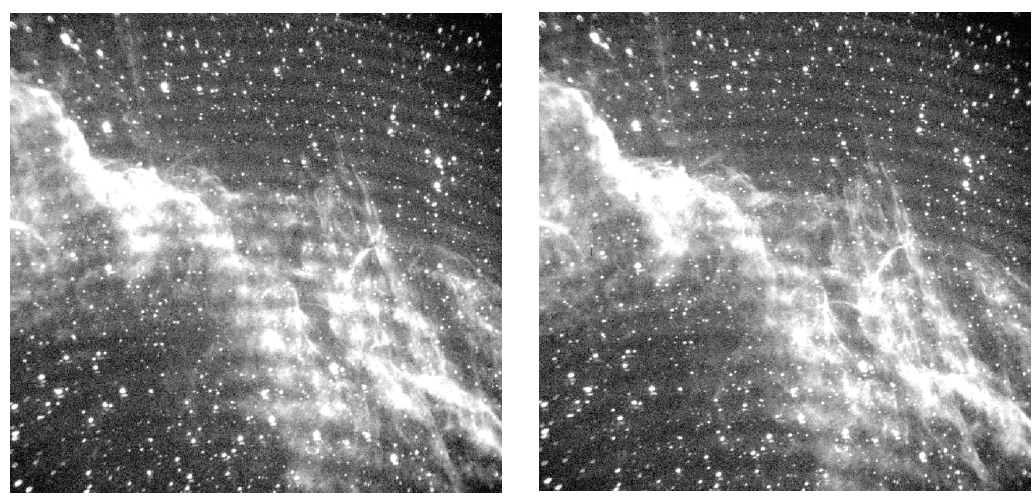

(a)

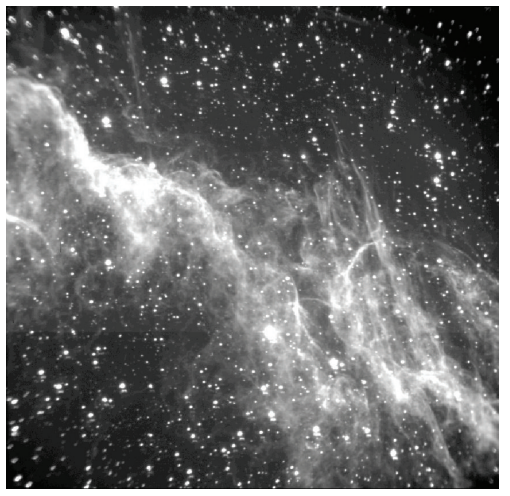

(b)

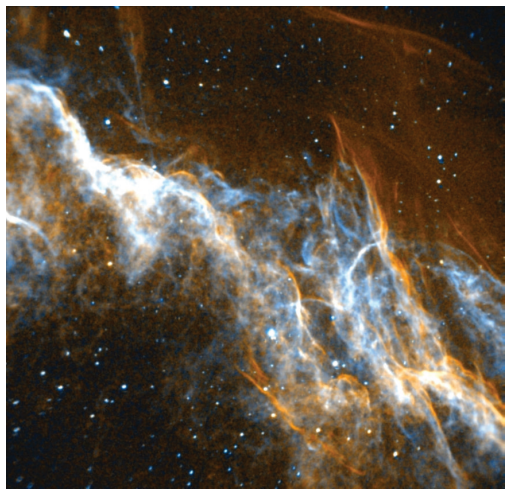

(c)

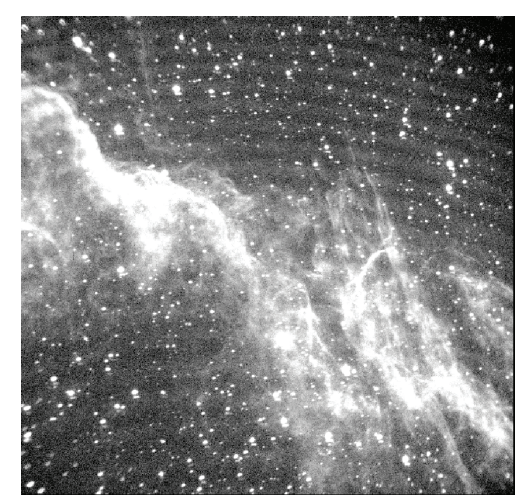


instrument favor the use of filters: the need to properly sample the interferogram to reach the required spectral resolution, and the distributed photon noise.

As mentioned above, the spectral resolution of a data cube is set by the maximum distance travelled by the moving mirror of the interferometer between the first and the last image of the cube. But one cannot simply take an image at a given mirror position and then move the mirror far from its initial position and hope to get a good resolution. The total optical path difference needs to be properly sampled, the step size being determined by the shortest wavelength and the total wavelength range to be covered by the cube. Using filters to restrict the total wavelength range and spectral folding (or aliasing) techniques allows increasing the mirror step length and thus the number of mirror steps for a given spectral resolution, at the expense of the total wavelength coverage. A simplistic example will help clarify this: say that we would like to use the bright lines $\mathrm{H} \beta$, [OIII] 4959, 5007, $\mathrm{H} \alpha$, [NII] 6548, 6584 and [SII] 6717, and 6731 to characterise an HII region. The minimum spectral resolution required in the red $(R \sim 1200)$ is set by the need to separate the [SII] doublet and in the blue $(R \sim 200)$ to separate $\mathrm{H} \beta$ from [OIII]. Getting $R=1200$ with an unfiltered cube covering the entire range allowed by a visible-band iFTS would require $\sim 1500$ mirror steps, which would limit the individual exposures to $\sim 6$ seconds for a 4-hour integration (taking the CCD readout time into account). Using two filters to isolate the blue and red line groups with the same spectral resolution requires only 120 steps and therefore allows much deeper individual images at each step for the same total time spent on the target.

The second reason to use filters is to reduce photon noise, caused by the well-known multiplex disadvantage of the FTS [16], which, in some cases, counterbalances its clear multiplex advantage. With a dispersive spectrograph, photon noise at a given wavelength is only due to the total number of photons (from the source and the sky) at this particular wavelength. But with an iFTS (this is also the case for traditional, singlepixel FTS), photons from the entire wavelength range allowed by the optics are detected at each step (wavelengths are not filtered, but modulated) and distributed equally amongst all wavelengths after the Fourier transform. In an iFTS cube of an HII region, photon noise from a bright [OI] 5577 nightsky line affects the much fainter nebular [NII] 5755 or HeI 6678 lines, which is not the case for a spectrum of the same object obtained with a dispersive spectrograph. This also explains why an iFTS is at its best targeting emission-line objects, for which the continuum is rather low compared to the strengths of the emission lines. In some cases (very low surface brightness features such as the ionized stripping tails in galaxy clusters, distant Ly $\alpha$ galaxies), the night sky continuum sets the detection limit.

Within the limits imposed by the compromise between spectral coverage and resolution, once a filter has been selected the user can choose its preferred spectral resolution, from $R=1$ (panchromatic image, which is anyway a byproduct of all data cubes) to the maximum limit imposed by the interferometer's architecture (typically a few times $10^{4}$ ), and tailor it for each object.
2.3. SpIOMM, an iFTS Prototype. In order to demonstrate the capabilities of a wide-field iFTS working in the visible band, our group has designed and built SpIOMM (Spectromètre Imageur de l'Observatoire du Mont Mégantic) at Université Laval [18] in close collaboration with ABB-Analytical (formerly Bomem), a Québec City-based company, and the Institut National d'Optique (INO). The primary objective of any astronomical instrument development being to address a science case, the design of SpIOMM was optimized to feed our science projects on the interstellar medium, late stages of stellar evolution, star formation, and galaxy evolution and which could not be obtained by existing instruments. This instrument, attached to the $1.6 \mathrm{~m}$ telescope of the Observatoire du Mont-Mégantic, is capable of obtaining seeing-limited, spatially resolved spectra of extended sources in filter-selected bandbases of the visible $(350-900 \mathrm{~nm})$ in a $12^{\prime} \times 12^{\prime}$ field of view with a spectral resolution $R \sim 10-25000$. It offers a very large contiguous field of view with $100 \%$ filling factor, resulting in millions of spectra per data cube. During its first few years of operations, only one output port was recorded, with a $1340 \times 1300,0.55^{\prime \prime}$-pixel LN-cooled CCD, with a readout time of 8 seconds. We recently added a $2 \mathrm{k} \times$ $2 \mathrm{k}$ CCD to its second output port.

SpIOMM's modulation efficiency is very good (85\%) in the red, as measured with nightly observations of a $\mathrm{He}-\mathrm{Ne}$ laser data cube, also used to spectrally calibrate the science cubes. The optical quality of its mirror and beamsplitter $(\lambda / 20$, peak-to-valley), as well as the prototype metrology system implemented, does not allow us to obtain exquisite modulation efficiency in the near UV $(\sim 25 \%)$, so most of our data cubes are obtained in the $450-700 \mathrm{~nm}$ range. Nevertheless, some cubes were obtained in the $U$ band, as shown in Figure 3.

Over the course of the past few years, we have obtained data cubes of HII regions [19], planetary nebulae [20], WolfRayet bubbles, and unique 3D views of young supernova remnants [21,22], as well as a sample of nearby spiral galaxies (next section). Exposure times vary from 7 seconds per step for the observations of bright nebulae in the red filter to 90 seconds per step for the observations of galaxies in the blue filter. A typical data cube therefore requires a total exposure time between one and five hours. The technical and scientific progress of SpIOMM have been described in a series of SPIE papers to which the reader is referred for more details [2326]. Applications of SpIOMM to the field of nearby galaxies are presented in the next sections.

\section{Nearby Spiral Galaxies: Emission Lines}

The new possibilities offered by iFTS to observe extragalactic HII regions will greatly improve our understanding of galaxy evolution. With a high spatial resolution, SpIOMM allows us to observe several hundred star forming regions simultaneously over all the structural components of a galaxy. The excellence of the statistics and the systematic characterization achieved for the HII regions will help to derive metallicity gradients and gain knowledge about the different mechanisms that drive star formation. In this paper, NGC628 data 


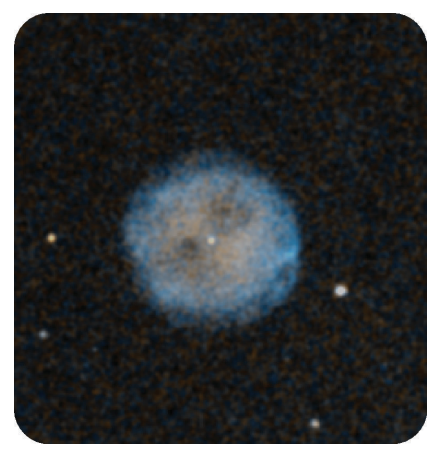

(a)

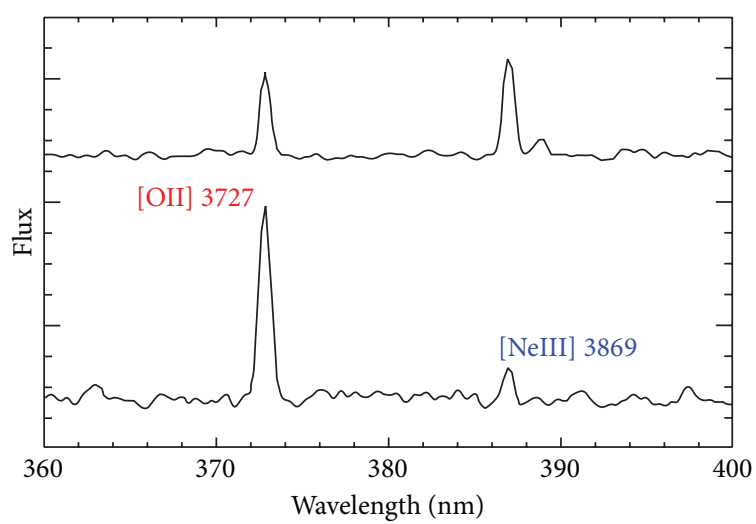

(b)

FIGURE 3: (a) Color-coded ([OII] 3727 in red, [NeIII] in blue) image of the planetary nebula M97, extracted from an SpIOMM data cube. (b) Spectra of selected pixels (binned $3 \times 3$ ) in the same cube with different [OII]/[NeIII] ratios.

cubes are going to be used as a typical example of SpIOMM's capabilities.

3.1. Observations and Data Reduction. Two filters have been used, a blue filter covering the wavelength domain from 475 to $515 \mathrm{~nm}$ and a red one from 650 to $680 \mathrm{~nm}$. They include several useful emission lines: $\mathrm{H} \beta$, [OIII] $\lambda \lambda 4959,5007$, [NIII] $\lambda \lambda 6548,6584, \mathrm{H} \alpha, \mathrm{HeI} \lambda 6678$, and [SII] $\lambda \lambda 6717,6731$. The spectral resolution $(R=\lambda / \Delta \lambda)$ of the data cubes is $\sim 700$ in the blue and $\sim 1600$ in the red. A binning of the pixels was performed to achieve a pixel size of $1.07^{\prime \prime}$. We applied the basic CCD corrections (bias, darks, and flats) to the data cubes before correcting for sky transparency variations. A $\mathrm{He}-\mathrm{Ne}$ laser was used for the wavelength calibration. The flux calibration was performed using data cubes of the standard star HD74721 in both filters. Finally, the average spectrum of the night sky was obtained from thousands of pixels around the galaxy and subtracted from the galaxy spectra (see Figure 4(a)).

3.2. Spectral Analysis. The ionized gas emission lines are fitted with two different techniques. One is based on a pixel-by-pixel Gaussian fit of spectral lines using the fithi routine from the IDL MAMDLIB library, available from the following web site: http://www.cita.utoronto.ca/ mamd/mamdlib.html. Figure 4(b) shows an example of the result for one pixel in an HII region.

The other method consists in using the HIIphot code [27] to define the HII regions contours and then fit the global spectrum (sum of the pixels in the same region) of each HII regions using the fithi routine. Figure 4(c) shows an example of an HII region contours identified with HIIphot over the $\mathrm{H} \alpha$ image. The parameters specified within HIIphot make sure that all the $\mathrm{H} \alpha$ flux is included in the HII region contour map.

At the time of writing, no correction has been applied yet for Balmer absorption lines. Long slit spectra obtained along the galactic radius are going to be used to correct for the impact of the absorption on $\mathrm{H} \beta$ and $\mathrm{H} \alpha$ lines. In the case of NGC628, absorption in $\mathrm{H} \beta$ and $\mathrm{H} \alpha$ is not negligible only in the very center of the galaxy (inner $1 \mathrm{kpc}$ ). For the moment with no correction for the absorption, the derived parameters using $\mathrm{H} \alpha$ line are really close to reality and for the parameters using $\mathrm{H} \beta$ line, we are only taking into account regions with radial position higher than $1 \mathrm{kpc}$. Detection limit for one pixel within an HII region is $\sim 6 \times 10^{-17} \mathrm{erg} \mathrm{s}^{-1} \mathrm{~cm}^{-2}$ in the $\mathrm{H} \alpha$ line. Figure 4(d) shows an example of a faint pixel spectrum.

3.3. Results. The internal dust extinction is calculated using the theoretical ratio $\mathrm{H} \alpha / \mathrm{H} \beta \approx 2.87$ for HII regions at $10000 \mathrm{~K}$ with $R_{V}=3.1$ (the Milky Way extinction on the line of sight was first taken into account). The internal $E(B-V)$ map of NGC628 is shown in Figure 5(a) using the pixel-bypixel analysis. The total $\mathrm{H} \alpha$ flux derived with SpIOMM for NGC 628 is $1.39 \times 10^{-11} \mathrm{erg} \mathrm{s}^{-1} \mathrm{~cm}^{-2}$. This value is in good agreement with other studies. For example, with a smaller field of view, Sánchez et al. ([28]; $\left.6^{\prime} \times 6^{\prime}\right)$ and Kennicutt et al. ([29]; $6.4^{\prime} \times 6.4^{\prime}$ ) found a value of $1.13 \times 10^{-11} \mathrm{erg} \mathrm{s}^{-1} \mathrm{~cm}^{-2}$ and $1.04 \times 10^{-11} \mathrm{erg} \mathrm{s}^{-1} \mathrm{~cm}^{-2}$, respectively, and with a larger field of view, Hoopes et al. $\left([30] ; 29^{\prime} \times 29^{\prime}\right)$ obtained a value of $1.51 \times 10^{-11} \mathrm{erg} \mathrm{s}^{-1} \mathrm{~cm}^{-2}$.

The dust-corrected pixel-by-pixel $\mathrm{H} \alpha$ flux map of NGC628 is shown in Figure 5(b). Its total dust-corrected $\mathrm{H} \alpha$ luminosity is then $3.56 \times 10^{41} \mathrm{erg} \mathrm{s}^{-1}$ (assuming a distance of 9.7 Mpc). The global star formation rate of NGC628, using Kennicutt's [31] formula (SFR $\left[\mathrm{M}_{\odot} \mathrm{yr}^{-1}\right]=7.9 \times 10^{-42} L_{\mathrm{H} \alpha}$ $\left[\mathrm{erg} \mathrm{s}^{-1}\right]$ ), is then SFR $=2.8 \mathrm{M}_{\odot} \mathrm{yr}^{-1}$. This is somewhat higher than the $2.4 \mathrm{M}_{\odot} \mathrm{yr}^{-1}$ estimated by Sánchez et al. [28] but the difference is explained by the larger number of HII regions revealed by SpIOMM in the external regions of the galaxy.

Using the $\mathrm{N} 2$ and the $\mathrm{O} 3 \mathrm{~N} 2$ metallicity indicators defined in [32] (PP04), we derived the metallicity of the HII regions identified with HIIphot as described above. As shown in Figure 6(a), a linear fit using the N2 indicator and all the HII regions identified gives the relation $12+\log (O / H)=$ $8.687-0.020 \mathrm{r}[\mathrm{kpc}]$. A very similar relation, $12+\log (\mathrm{O} / H)=$ $8.689-0.022 \mathrm{r}[\mathrm{kpc}]$, is obtained using the $\mathrm{N} 2$ indicator for a subsample of HII regions also characterized with the O3N2 indicator (the $4 \sigma$ detections of the OIII $\lambda$ [5007] 

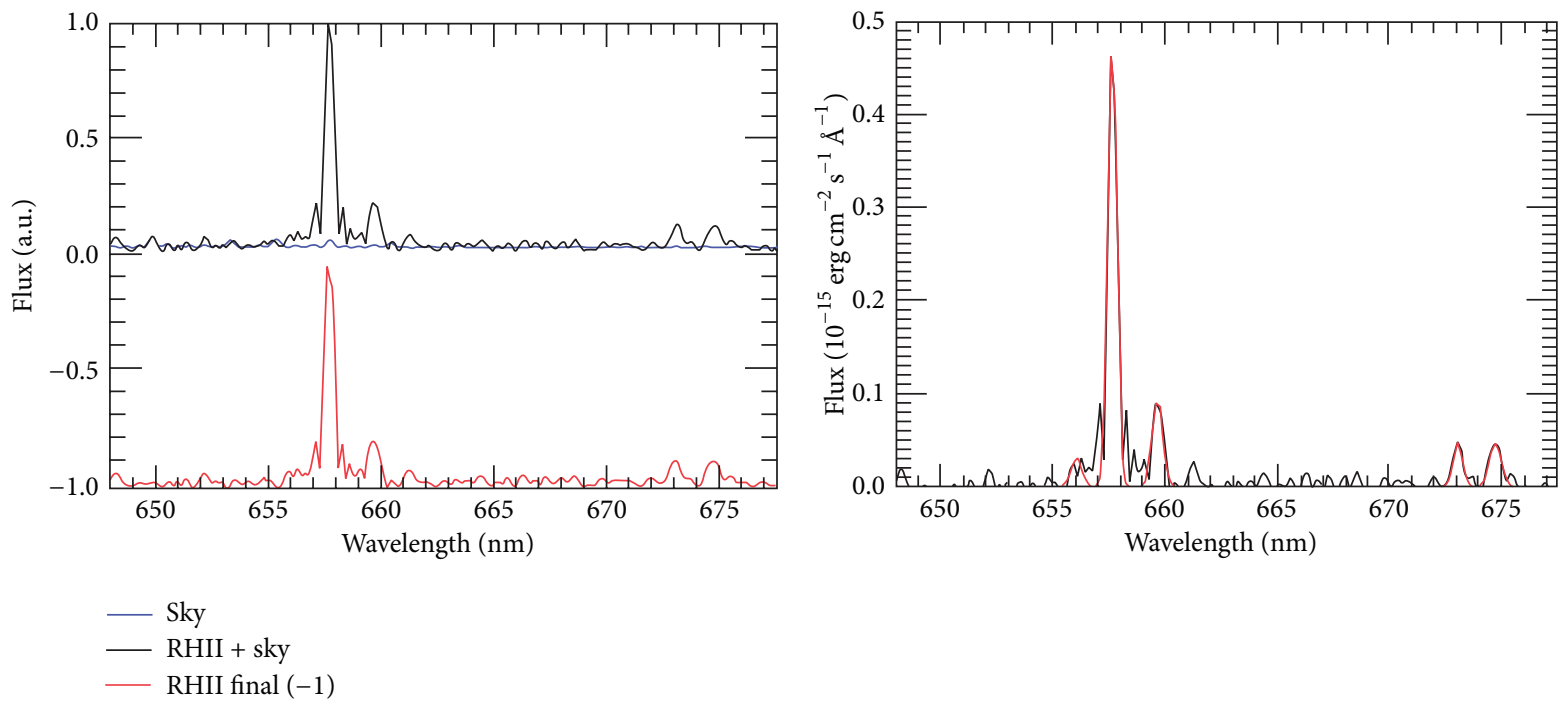

(a)

(b)

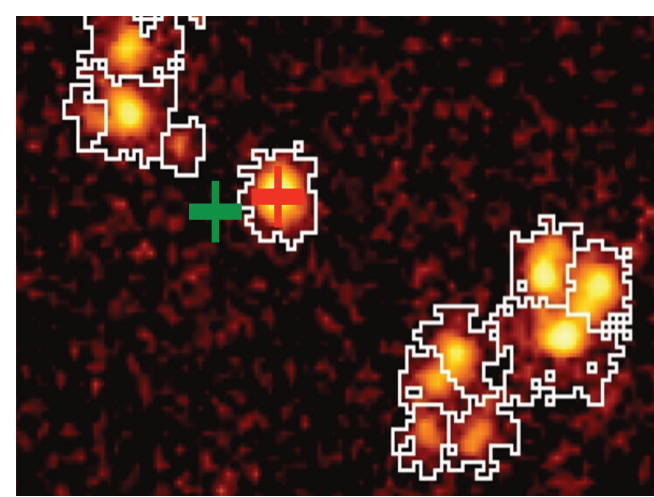

(c)

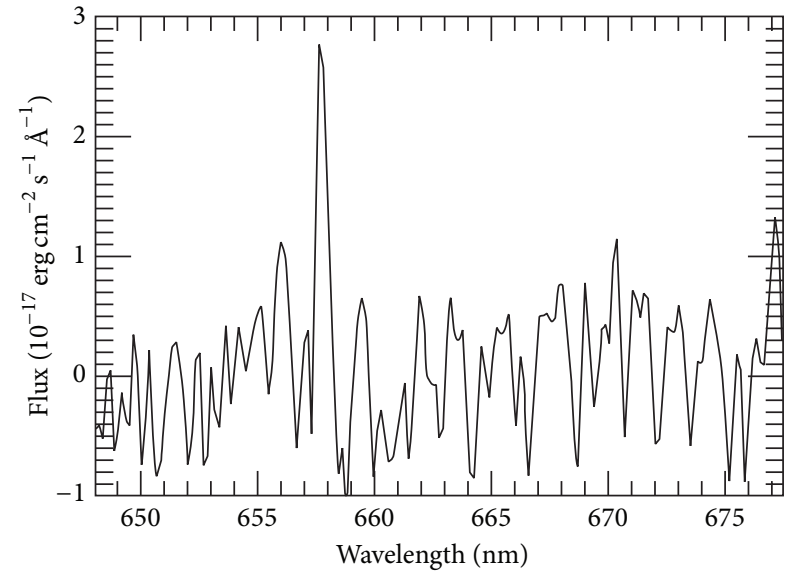

(d)

Figure 4: (a) Example of the sky subtraction for the spectrum of a bright pixel (1h $\left.36 \mathrm{~m} 54.1 \mathrm{~s} ;+15^{\circ} 46 \mathrm{~m} 10.8 \mathrm{~s}\right)$. (b) Example of a Gaussian fit on the spectrum of a bright pixel. (c) Close-up on the contours map of NGC 628 selected by HIIphot; the field of view is $1.5^{\prime} \times 1^{\prime}$. The red cross identifies the position of the bright pixel used for the previous sky subtraction and Gaussian fit examples. The green cross identifies the pixel with $\mathrm{H} \alpha$ in emission at $\sim 2 \sigma$ limit of detection. (d) Example of the spectrum of a $1.1 \operatorname{arcsec}^{2}$ pixel with $\mathrm{H} \alpha$ in emission at $\sim 2 \sigma$ limit of detection.

lines have been successful for only 134 regions). As shown in Figure 6(b), if the $\mathrm{O} 3 \mathrm{~N} 2$ indicator is used, we find a steeper gradient, $12+\log (\mathrm{O} / H)=8.780-0.033 \mathrm{r}[\mathrm{kpc}]$. Contrary to the $\mathrm{O} 3 \mathrm{~N} 2$ indicator, the $\mathrm{N} 2$ indicator has a local maximum in its metallicity distribution [33] that, we suspect, may introduce more dispersion and uncertainty in the N2 gradient.

A big advantage of an instrument like SpIOMM is the possibility to perform a detailed spatial analysis of the emission lines. The emission lines studied are affected by changes in the ionization parameter and the electron temperature [33] as we move away from the HII regions. Furthermore, a component in emission may come from the galaxy diffuse ionized gas (DIG; [34]). The origin for the DIG is still complex (background ionization from older stellar populations, stellar winds, AGN, shocks). Among others, the DIG can contribute to low ionization species, like NII, SII, $\mathrm{H} \alpha, \mathrm{H} \beta$, and OII $[35,36]$. In NGC628, we see systematic changes in line ratios as a function of the distance from an $\mathrm{H} \alpha$ emission peak: in the case of a relatively low metallicity $(\sim 8.4)$ region we find an increasing $[\mathrm{NII}] \lambda 6584 / \mathrm{H} \alpha$ ratio and a decreasing $[\mathrm{OIII}] \lambda 5007 / \mathrm{H} \beta$ ratio (see the examples in Figure 7 for NGC $628)$, while for a relatively high metallicity $(\sim 8.8)$ region, these line ratio variations are inverted (see the examples in Figure 8 for NGC 628). These behaviors are to be discussed in terms of the DIG contribution and variation in the HII region conditions, but they clearly point out here to the importance of the spatial resolution when defining and characterizing HII regions and observing accurate metallicity gradient. As shown by the pixel-by-pixel O3N2 metallicity map of NGC 628 (Figure 9), if one uses a larger aperture to study the HII regions, it will have an impact on the abundance estimate for 


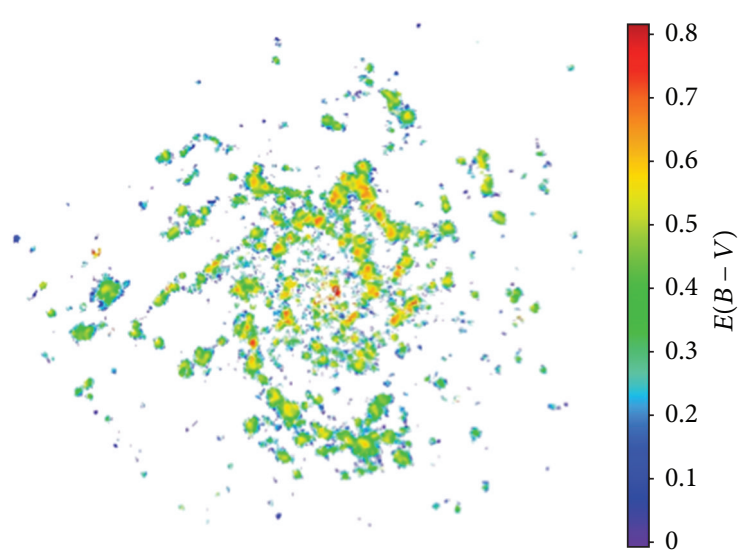

(a)

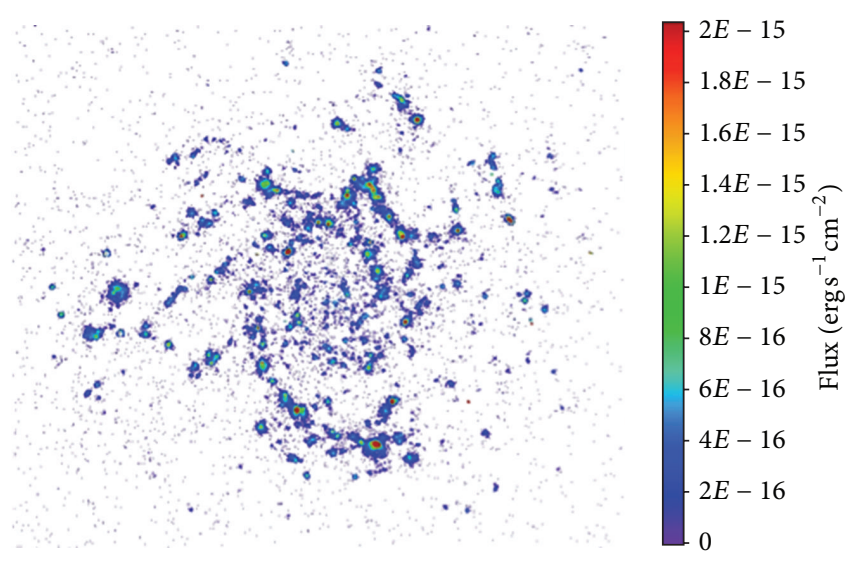

(b)

FIGURE 5: (a) NGC 628 dust extinction map from the $\mathrm{H} \alpha / \mathrm{H} \beta$ ratio. (b) NGC 628 dust-corrected $\mathrm{H} \alpha$ flux map. Pixel size is 50.5 parsec ${ }^{2}$ for a distance of 9.7 Mpc. The field of one CALIFA pointing is shown in the top right corner.

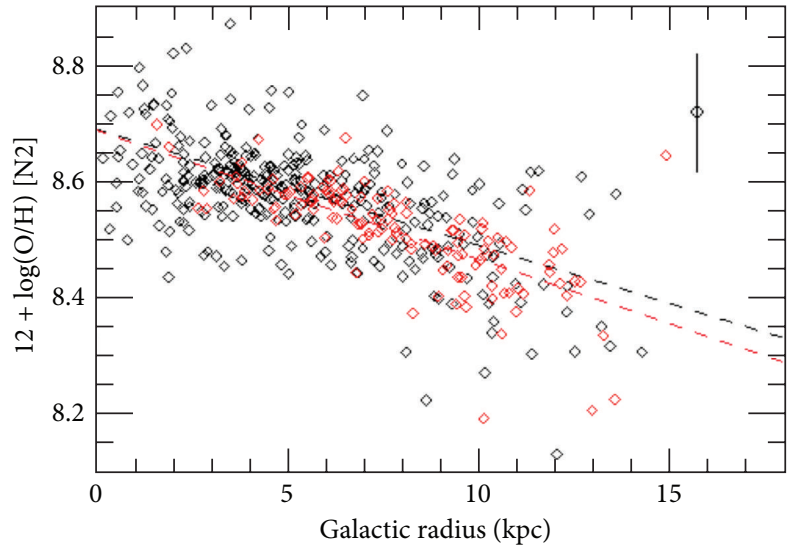

(a)

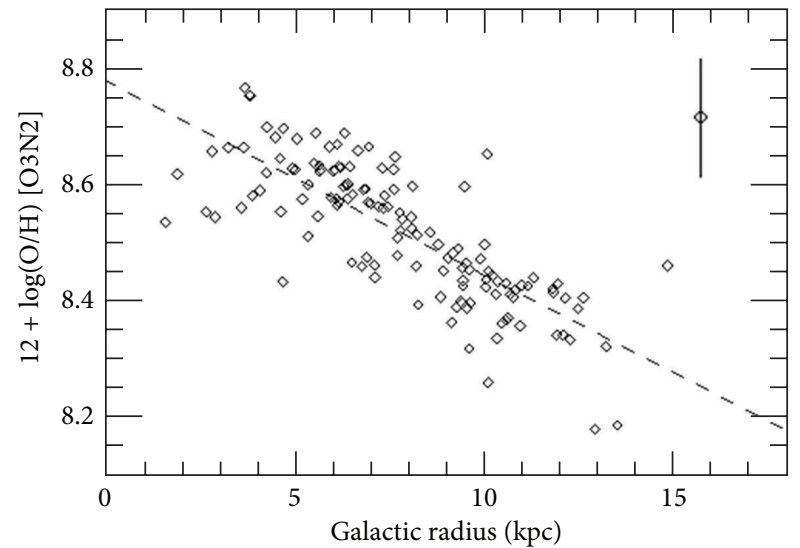

(b)

FIGURE 6: (a) Metallicity gradient of NGC628 derived using the N2 indicator from PP04 and the HII regions defined with HIIphot. The black diamonds (and their corresponding linear fit given by the black dash curve) is obtained using all the HII regions identified. The red diamonds (and corresponding dash red linear fit) are for the 134 regions that can also be characterized with the O3N2 indicator. (b) Metallicity gradient derived using the O3N2 indicator from PP04 and 134 HII regions defined with HII phot. Typical error bars are shown on the plot.

each region and also on the galaxy metallicity gradient. DIG effects on the characterization of extragalactic HII regions are going to be discussed in future publications.

\section{Elliptical Galaxies: Absorption Lines}

4.1. Absorption Lines Extraction. The vast majority of commercially available FTS use this technique to measure the strength of absorption lines in their target spectra. So nothing in the iFTS concept prevents it from obtaining spectra of continuum sources with absorption features. However, the multiplex disadvantage discussed above increases the noise level for a given spectral element compared to dispersive spectroscopy. This distributed noiserecorded in the interferogram (photon noise from the entire bandpass contributes to the recorded signal at each mirror step) is transferred to the spectrum after the FFT.
Figure 10 shows a simplified example of distributed noise. Given two sources of equal total flux, one with emission and one with absorption, it is clear that the interferograms are utterly different even if the mean value is the same. The emission line shows large variation in its interferogram while they fade quickly for the absorption line. In this example, only a $\sqrt{N}$ photon noise is applied and, since the mean flux is the same for both lines, the average level of noise is about the same. This in turn produces a level of noise in the spectra that is also about the same for both. It is then easy to see that if one were to extract the profile of both lines, the level of noise compared to the flux in the line would be much higher for the absorption feature. For the same mean flux in the bandwidth covered by the filter, spectra dominated by emission lines will show a greater SNR than spectra of continuum sources with absorption lines. Stellar spectra have been nevertheless obtained on a regular basis with SpIOMM; some are shown 


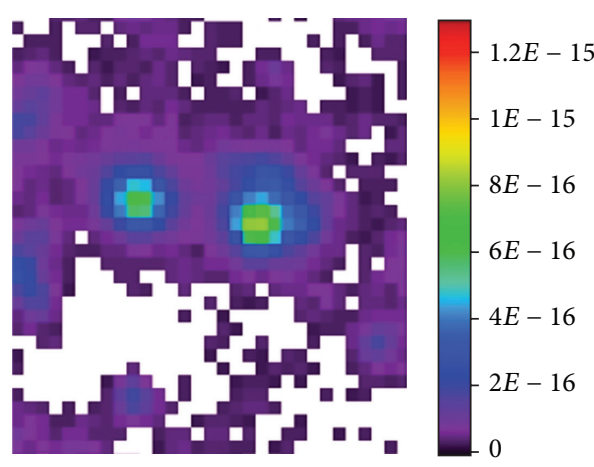

(a)

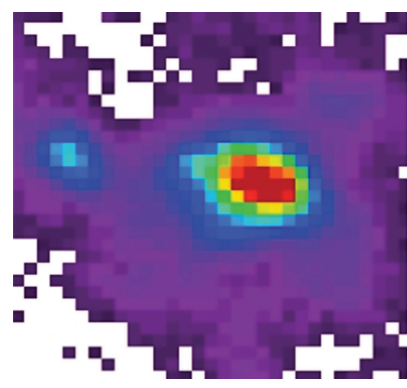

(d)

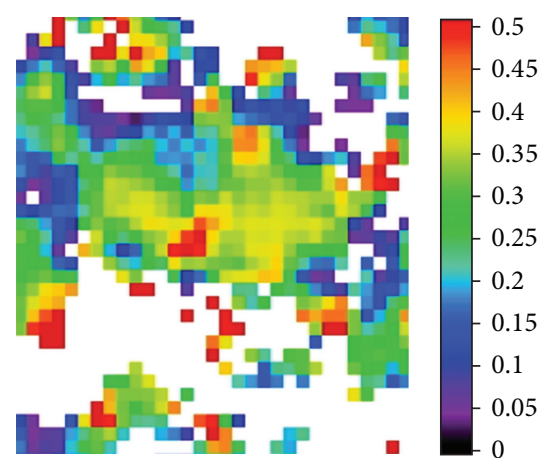

(b)

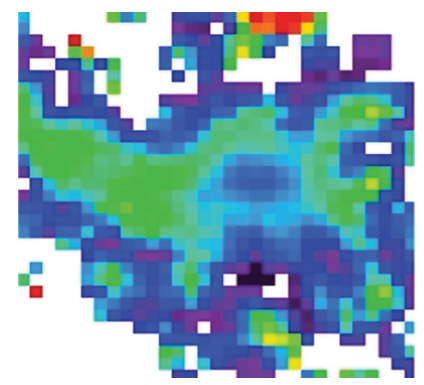

(e)

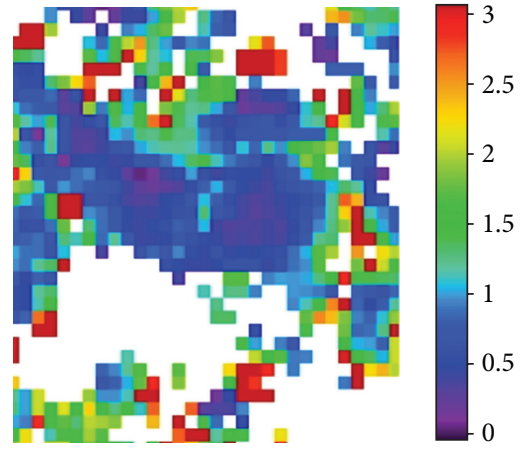

(c)

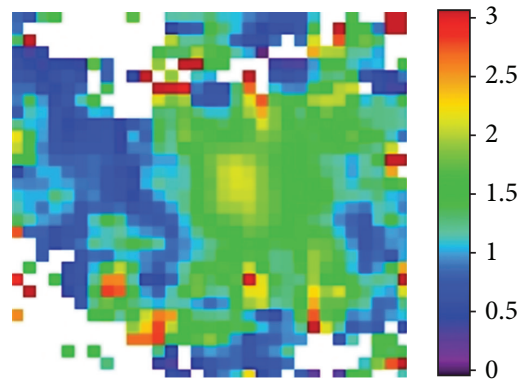

(f)

Figure 7: Images of HII regions located at RA $=1 \mathrm{~h} 36 \mathrm{~m} 38.9 \mathrm{~s}, \mathrm{DEC}=15 \mathrm{~d} 44 \mathrm{~m} 24.8 \mathrm{~s}$ with $12+\log (\mathrm{O} / \mathrm{H}) \sim 8.4$ ((a), (b), and (c)), and at $\mathrm{RA}=1 \mathrm{~h} 36 \mathrm{~m} 38.4 \mathrm{~s}, \mathrm{DEC}=15 \mathrm{~d} 47 \mathrm{~m} 00.9 \mathrm{~s}$ with $12+\log (\mathrm{O} / \mathrm{H}) \sim 8.8((\mathrm{~d})$, (e), and (f)). In both cases, the image on the left shows the H $\alpha$ maximum intensity $\left[\mathrm{erg} \mathrm{s}^{-1} \mathrm{~cm}^{-2}\right.$ ], center: $[\mathrm{NII}] \lambda 6584 / \mathrm{H} \alpha$, right: $[\mathrm{OIII}] \lambda 5007 / \mathrm{H} \beta$. FOV is $33^{\prime \prime} \times 33^{\prime \prime}$, North at the top, East to the left.

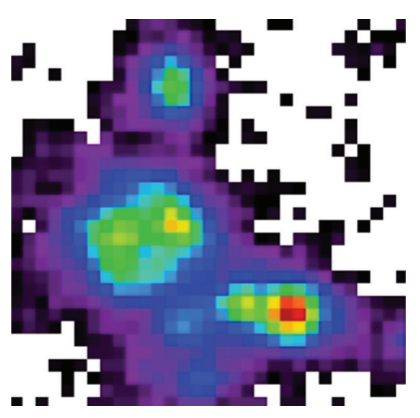

(a)
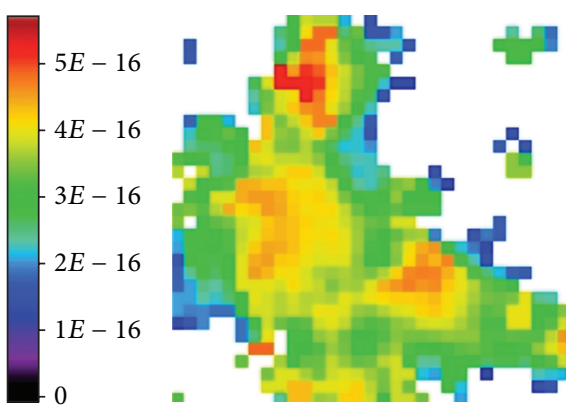

(b)

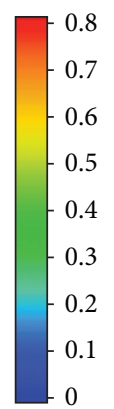

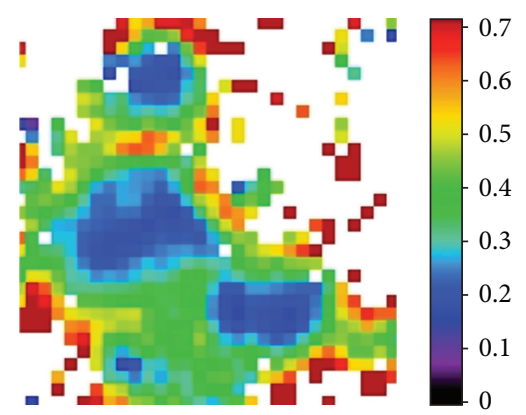

(c)

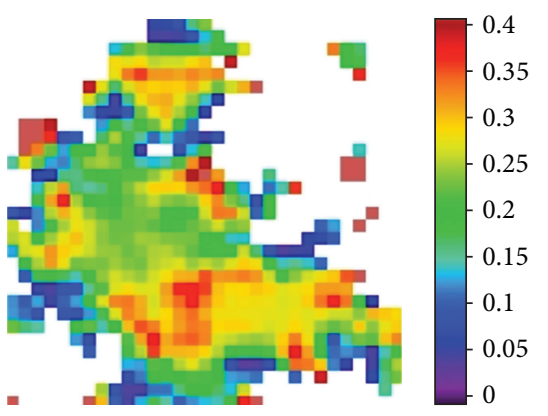

(d)

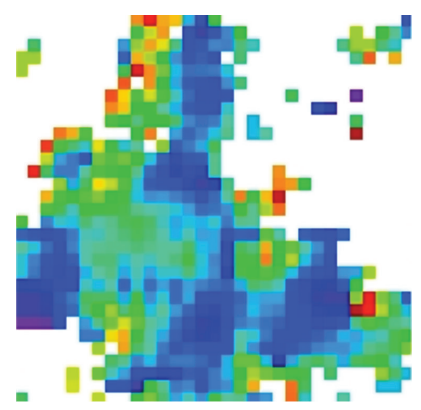

(e)
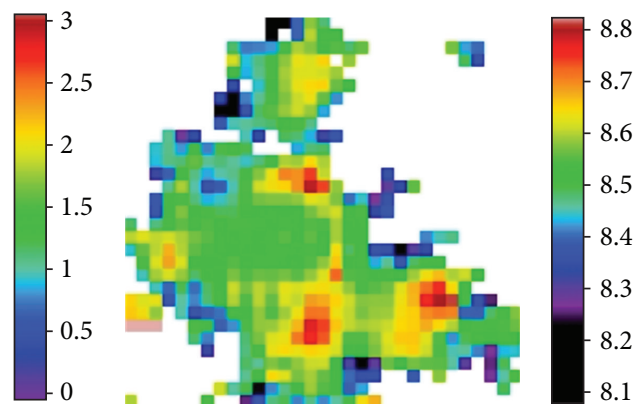

(f)

FIGURE 8: Images centered on HII regions located around RA $=1 \mathrm{~h} 36 \mathrm{~m} 46.5 \mathrm{~s}, \mathrm{DEC}=15 \mathrm{~d} 48 \mathrm{~m} 56.8 \mathrm{~s}$. Top left: H $\alpha$ maximum intensity [erg s ${ }^{-1} \mathrm{~cm}^{-2}$ ], top center: $E(B-V)$, top right: [SII] $\lambda \lambda 6717+6731 / \mathrm{H} \alpha$, bottom left: [NII] $\lambda 6584 / \mathrm{H} \alpha$, bottom center: $[\mathrm{OIII}] \lambda 5007 / \mathrm{H} \beta$, and bottom right: O3N2 indicator from PP04. FOV is $33^{\prime \prime} \times 33^{\prime \prime}$, North at the top, East to the left. 


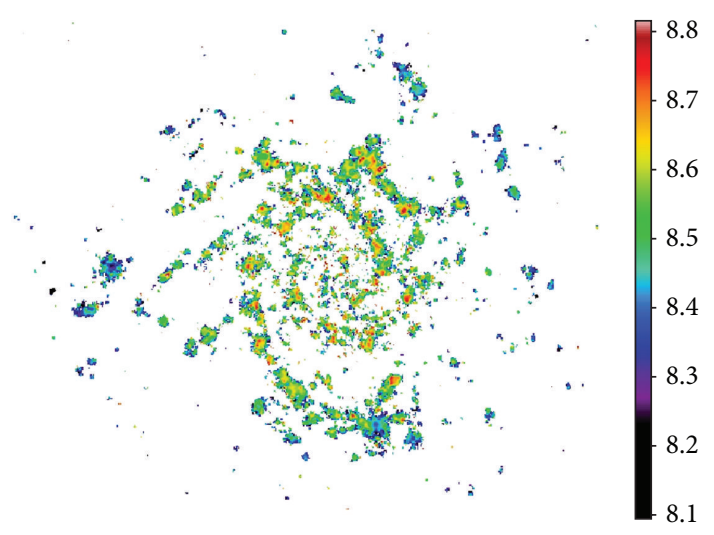

FIGURE 9: Pixel-by-pixel metallicity map using O3N2 indicator from $\mathrm{PP} 04$. FOV is $12^{\prime} \times 12^{\prime}$.

in Figure 11. In fact, all SpIOMM data cubes include stars for which spectra can be extracted.

It is then obvious that an iFTS is not the ideal instrument to observe individual stars. However, the wide field of view provided by SpIOMM allows observations of hundreds of stars simultaneously, as well as extended absorption line sources, which compensates for the multiplex disadvantage. Cubes of open clusters were obtained, and a technique was developed to optimize the extraction of the stellar interferograms, taking into account seeing variations during the cube acquisition; results will be presented elsewhere.

4.2. The Case of M87. To quantify SpIOMM's ability to provide scientifically useful absorption spectra of an extended source, we targeted the giant elliptical galaxy M87 using SpIOMM's custom V filter (538-649 nm). Our primary goal was to compare SpIOMM's data with those obtained from direct imagery and long-slit spectroscopy. Using IRAF's STSDAS package, we first extracted luminosity profiles and isophotes from the panchromatic image obtained by combining all slices from the spectral cube (see Figure 12(a)). Our data being in excellent agreement with multiband imagery from Liu et al. [37], we then extracted spectra along isophotal lines at different galactocentric radii, shown in Figure 12(b). The spectra show many interesting features, the two most important being a molecular $\mathrm{TiO}$ band on the red side and the sodium doublet line (NaD) in the center near $5900 \AA$.

In order to compare our data with those obtained by Davidge [17] with a long-slit spectrograph at the CanadaFrance-Hawaii $3.6 \mathrm{~m}$ Telescope, we extracted the NaD index as defined by Worthey and Ottaviani [38] as well as this line's equivalent width determined from a fitted Gaussian profile. These values are compared in Figure 13, which shows a clear negative gradient with increasing galactocentric radius. Our Lick $\mathrm{NaD}$ index values are slightly lower than Davidge's, on average, but this is mostly due to our lower spectral resolution (1.2 nm compared to $0.6 \mathrm{~nm})$. Correction suggested by Vazdekis et al. [39] to compensate for this would add about $0.4 \AA$ to our values which would bring them closer to Davidge's but would not affect the slope of the gradient which is similar.
The extraction of scientific data from absorption features in an extended object like a galaxy is proving to be feasible. Both visual and spectral data obtained on the source with the imaging FTS SpIOMM are in good agreement with the literature. Stability is of the essence during the observation to ensure the best possible SNR on the absorption features. This is particularly important for SITELLE, which should enable much more detailed and precise study of absorption features due to its more stable design compared with SpIOMM.

\section{Next Steps: SITELLE and SNAGS}

5.1. SITELLE. SpIOMM has been a wonderful prototype to work with and to learn from, special thanks to our privileged access to the well-equipped $1.6 \mathrm{~m}$ telescope at the Observatoire du Mont Mégantic, where observing conditions can be harsh, especially in winter when temperatures can drop to $30^{\circ} \mathrm{C}$. But the full advantages of the iFTS technology can only be attained with a more robust instrument installed on a large telescope with excellent sky conditions. Our team therefore designed and built SITELLE, an iFTS accepted as a guest instrument by the Canada-France-Hawaii Telescope (CFHT). All the lessons learned from our regular use of SpIOMM have been implemented in the design of SITELLE [40, 41] and its data reduction software, ORBS [42]. SITELLE's field of view and maximum spectral resolution (resp., $11^{\prime} \times 11^{\prime}$ and $R \sim$ 25000) are very similar to SpIOMM's, but its performance will be greatly enhanced, especially in the near UV, thanks to very high quality optics within the interferometer cavity $(\lambda / 30)$, more robust metrology, servo mechanism, and structural stiffness leading to very low mirror jitter ( $10 \mathrm{~nm}$ RMS), as well as high QE, and low read noise $2 \mathrm{k} \times 2 \mathrm{k} C \mathrm{CD}$ s by e $2 \mathrm{v}$. Thanks to four readout amplifiers on each CCD, readout time is $\sim 3 \mathrm{~s}$, thereby increasing the overall efficiency of the instrument. The overall throughput of SITELLE, including modulation efficiency and detectors' quantum efficiency, is shown in Figure 14. Spatial sampling is also improved $\left(0.32^{\prime \prime}\right.$ pixels) to match the excellent seeing provided by the CFHT. Sky brightness on Mauna Kea is also remarkably low, significantly improving the detectability of faint sources. If all these factors are taken into account, we estimate that SITELLE at the CFHT will be about 15 times more sensitive at $350 \mathrm{~nm}$ and 6 times more at $700 \mathrm{~nm}$ than SpIOMM at OMM.

At the time of writing, SITELLE is in the final phases of integration and testing, for a planned delivery at the CFHT in early 2014. First light, commissioning, and science verification will occur soon after. As a guest instrument, SITELLE will be accessible to all CFHT partners. The long list of projects presented at a workshop organized in May 2013 (http://www.craq-astro.ca/sitelle/talk.php) has demonstrated the interest of the CFHT community (and beyond) for this instrument in areas as diverse as comets, star clusters, nearby galaxies, and high redshift Lyman- emitters. As demonstrated by Graham et al. [43] in a paper describing the rationale for equipping a space-based IR telescope with an iFTS, such an instrument is a very powerful tool to study distant galaxies in a relatively unbiased way. At very low flux levels, sky background sets the detection limits of emission-line 


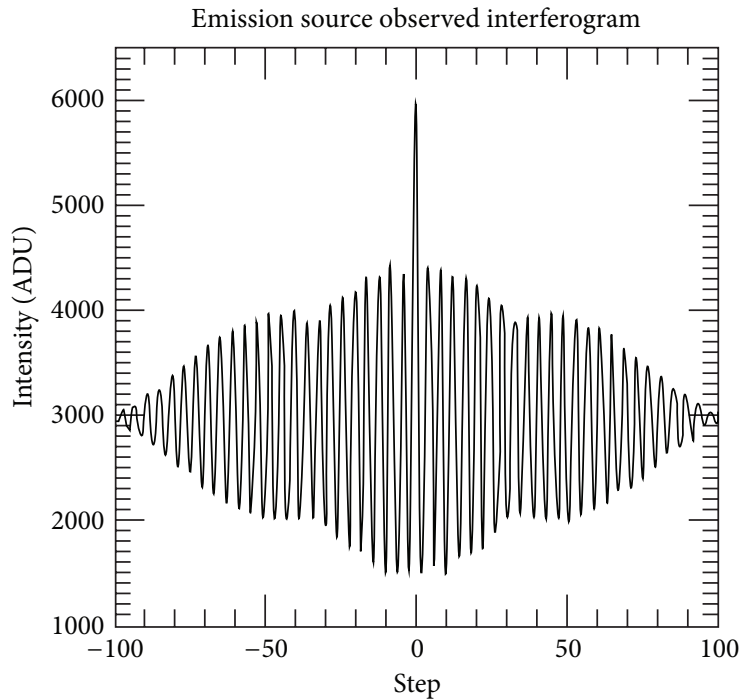

(a)

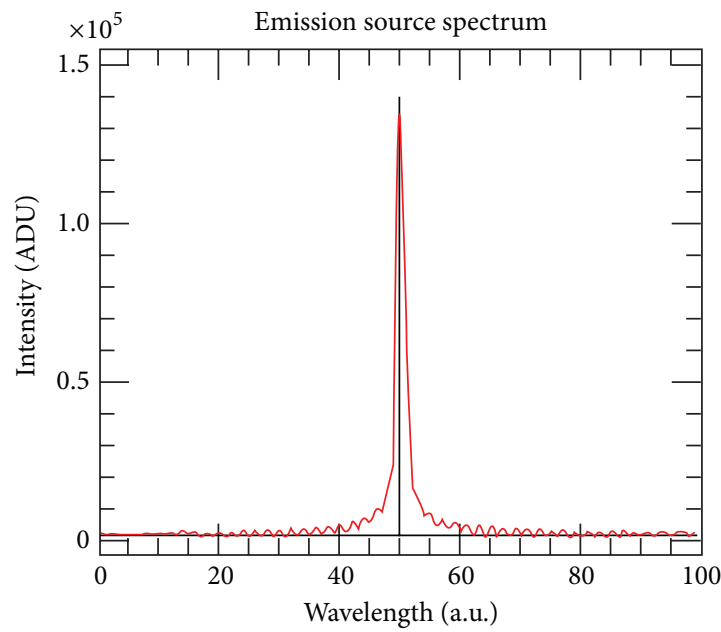

(c)

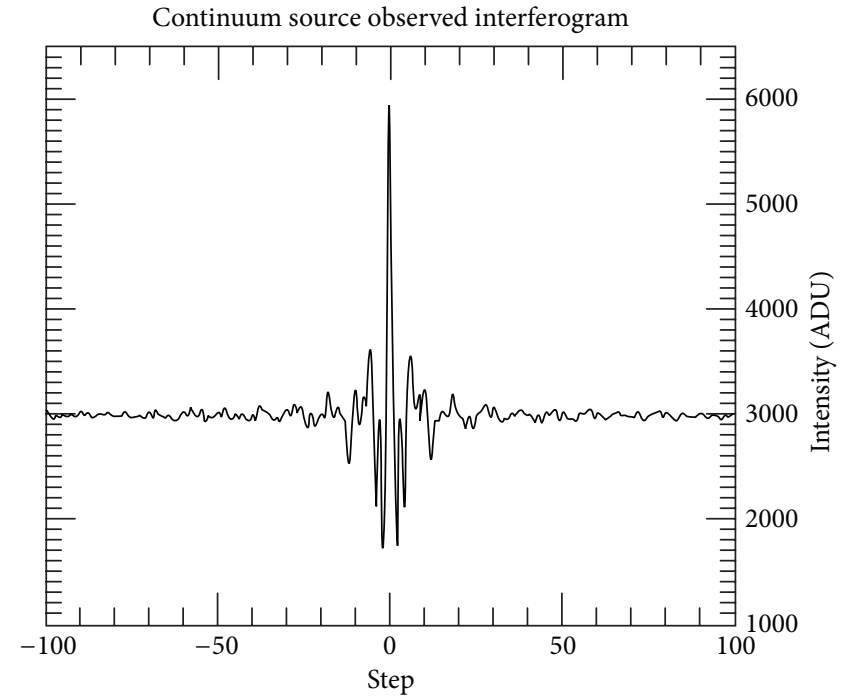

(b)

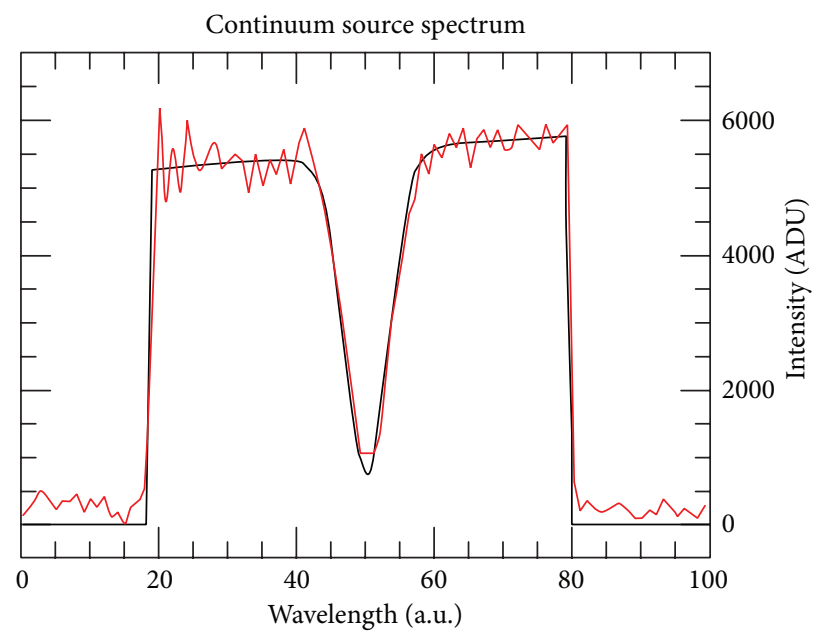

(d)

FIGURE 10: Comparison between simulated emission and absorption lines spectra.

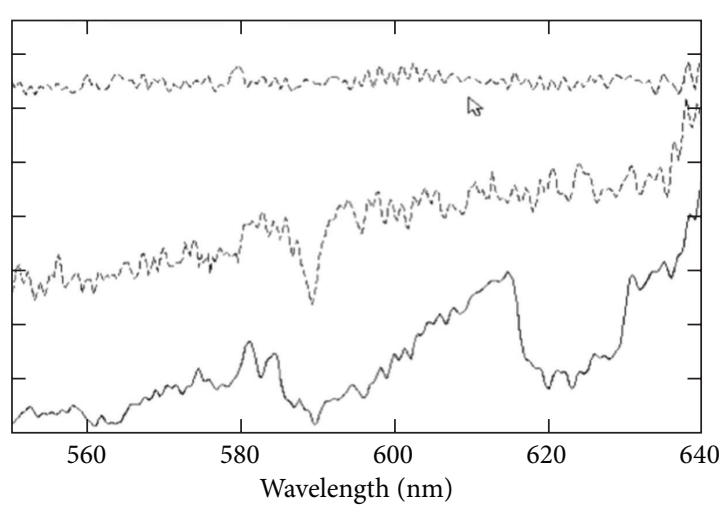

(a)

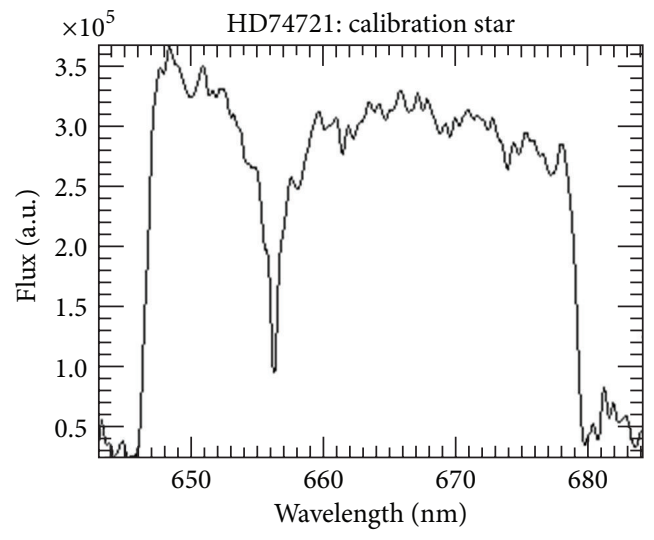

(b)

FIGURE 11: (a) Spectra of the 15th magnitude stars in the field of the planetary nebula M27; from top to bottom: the central white dwarf (featureless, as expected, in this wavelength range), a solar-type star with the $\mathrm{NaD} 5890,96$ lines, and an $\mathrm{M}$ star showing TiO bands. (b) Spectrum of the standard horizontal branch A-star HD 74721 used to flux-calibrate the NGC 628 data cube (see above). 


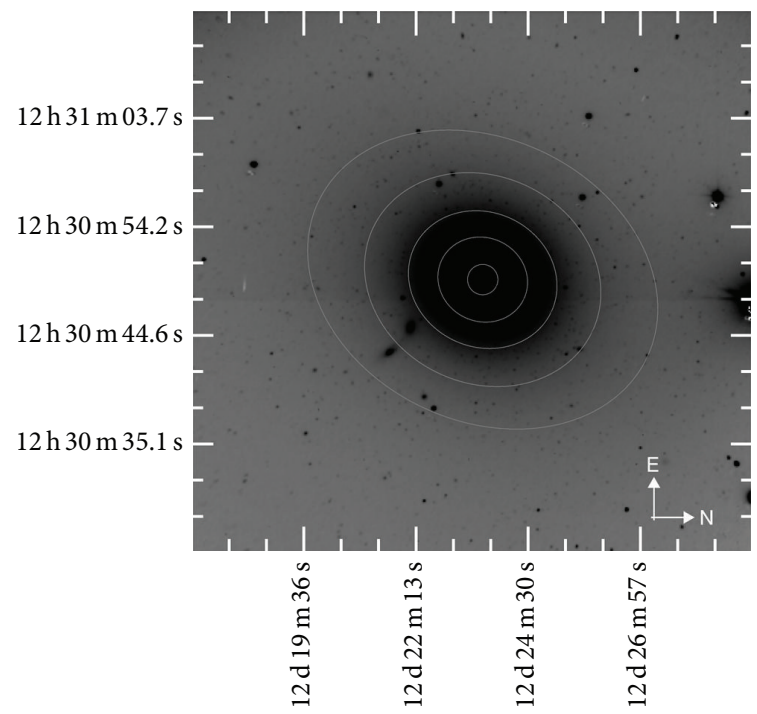

(a)

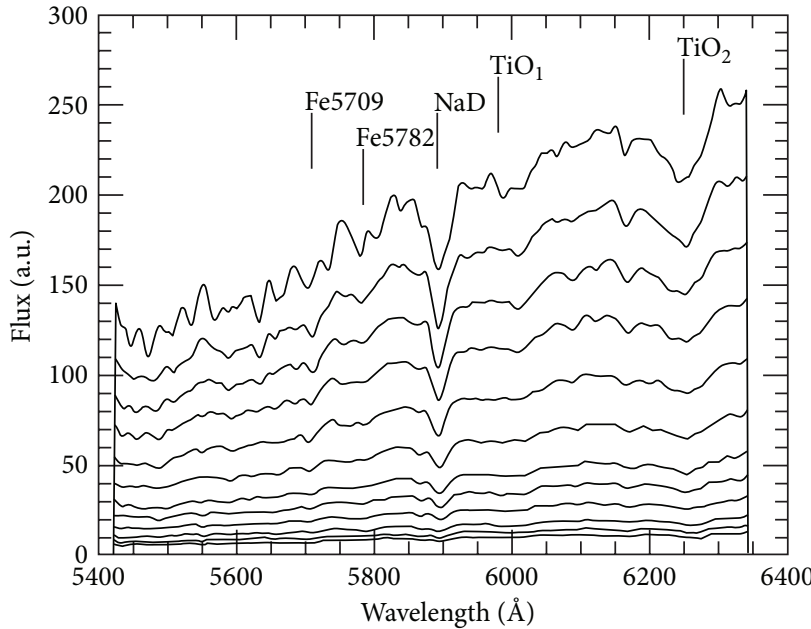

(b)

Figure 12: (a) Deep panchromatic V-band image of M87 extracted from the data cube showing some isophotes used to extract spectra. (b) Deredshifted radial spectra of M87. From top to bottom, $\log r(\operatorname{arcsec})=0.79,0.93,1.06,1.17,1.25,1.35,1.46,1.54$, and 1.61.

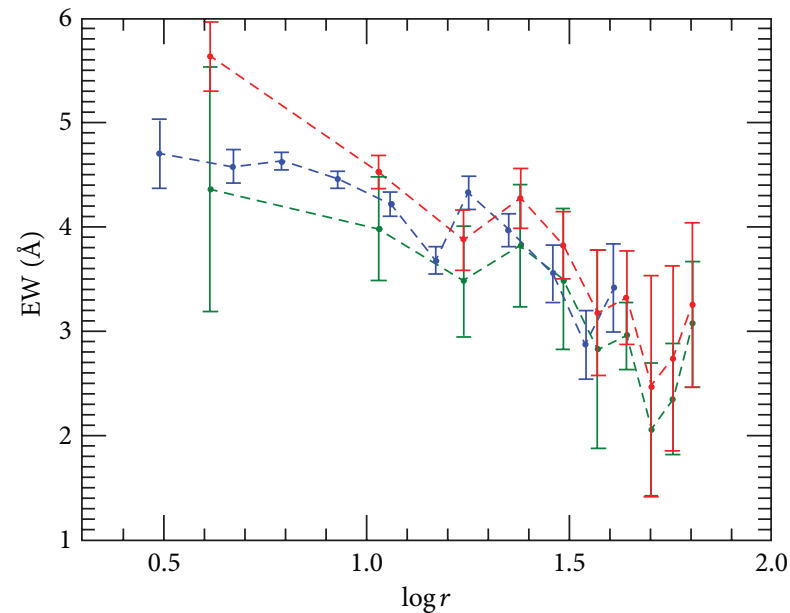

-. NaD calculated lick indices

- NaD spectroscopic EW

- NaD lick indices from Davidge 1992

FigURE 13: Strength of the NaD absorption line in the spectra of M87 at different galactocentric radii obtained from the SpIOMM data cube with two different methods and compared with Davidge [17].

sources; visible-band sky background on Mauna Kea being very similar to that of IR background in space, SITELLE can be seen as the ground-based version of Graham's proposed iFTS. The power of SITELLE in this type of study is that it will sample the redshift space uniformly on a wide field, allowing unbiased spectroscopic redshift determination and line profile analysis on several galaxies per cube. In order to follow up on the SpIOMM work presented in this paper,

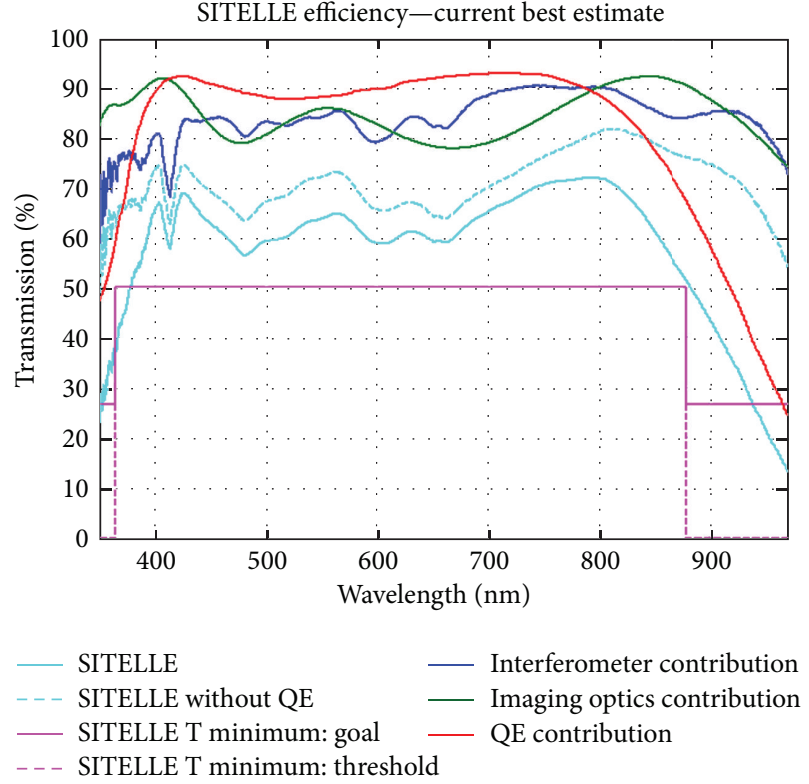

FIGURE 14: SITELLE's expected combined throughput, including modulation efficiency.

the next section summarizes the plans for a survey of nearby galaxies, SNAGS.

5.2. SNAGS. Powerful constraints on models of galactic evolution and the dynamical processes that transform galaxies are derived from homogeneous determinations of chemical abundances in individual gaseous nebulae, the distribution of their stellar populations in terms of age and metallicity, 
and the gaseous and stellar kinematics. With its efficient and versatile $3 \mathrm{D}$ spectroscopic capabilities over a large field-ofview, SITELLE offers an opportunity to study these evolution constraints in a significant number of large, nearby disk galaxies. Recent examples of using integral field capabilities to study nearby disk galaxies show the full potential of this technique to assess nebular abundances and stellar metallicity [28]. We are currently planning to a conduct a survey (SNAGS) of a sample of $\sim 75$ large $\left(D>3^{\prime}\right)$, nearby spirals with SITELLE at CFHT. Our main objectives are (1) to determine the spatial distribution of nebular abundances across their disk and assess radial, azimuthal, and local variations within different galaxy components (i.e., bars, arm and interarm regions, and outer disk), (2) to reconstruct their star formation history through stellar populations, and (3) to map the gaseous and stellar kinematics and study the diverse dynamical processes governing large-scale star formation and mixing within their disks.

As demonstrated by other papers in this volume, SNAGS is not the first project aiming at integral field spectroscopy of a large sample of nearby galaxies. One of the earliest and most successful is the SAURON survey, mostly concentrating on early-type galaxies $[44,45]$. But by far the most similar programs are CALIFA [46-48] and VENGA $[49,50]$. The science objectives of SNAGS will be distinguished from those of the other two projects by the main advantages of its instrumental setting: significantly higher spatial resolution (limited only by CFHT's exceptional image quality and SITELLE's pixel size of $0.32^{\prime \prime}$ ) with $100 \%$ filling factor; higher spectral resolution $(R \sim 2000)$ which, combined with a very precise wavelength calibration inherent to the iFTS concept, will allow detailed kinematics studies on very small scales; very wide field of view which, combined with SITELLE's high throughput from the near UV across the visible range and Mauna Kea's dark sky, will allow us to probe the outermost regions of galaxies.

Our preliminary sample of galaxies includes objects of different morphologies, masses, and environment. For instance, several objects with bars will be observed to investigate the potential role of nonasymmetrical components in triggering/quenching star formation and in mixing chemical elements in galaxy disks. Other objects with star formation in their very outer disks (e.g., identified from GALEX observations) were selected to evaluate large-scale breaks in radial abundance gradients and to study chemical processes at the periphery of these disks [51]. SNAGS will obtain spectrophotometrically calibrated data cubes within three spectral ranges to cover the strong lines from [OII] $\lambda 3727$ to $[\mathrm{SII}] \lambda \lambda 6717,6731$. Nebular abundances will be determined using several line-ratio methods and calibrations (e.g., $\mathrm{R}_{23}$, N2Ha, and O3N2, see Kewley and Zahid [52] for a review);complementary observations on larger telescopes will be obtained for a smaller sample of galaxies to derive the abundances through "direct" auroral line measurements in order to evaluate the "indirect" methods. Gas kinematics on a small-scale will be studied using moderate resolution $(R \sim 4000)$ for all three spectral ranges. Current studies so far (e.g., CALIFA and VENGA) have concentrated on global properties (abundance gradients) in galaxies. SNAGS will also assess small-scale variations caused by multiphase stellar winds, enrichment by starburst clusters in peculiar evolutionary stages, and dynamical processes. SITELLE is an ideal instrument to concentrate on these small-scale $(<100 \mathrm{pc})$ variations and establish conditions under which they take place. A pilot study for SNAGS will be carried out during the science verification phase for the instrument planned for early 2014, with the survey itself starting later on after final commissioning.

\section{Conclusions}

We have presented in this paper the iFTS as a viable approach to integral field spectroscopy. The regular use of SpIOMM and preliminary tests performed with SITELLE have demonstrated that the technical challenges imposed by the very stringent requirements on the optical quality of an iFTS components as well as its metrology and servo system have been overcome and now they allow us to reach a high modulation efficiency in the visible and the near UV.

The science niche for the iFTS approach must take into account its main advantages (very wide field of view, high throughput, seeing-limited image quality, and flexible spectral resolution) and disadvantages (spectrally distributed noise, and necessary compromise between spectral coverage and resolution) compared with standard integral field spectrographs. Although the original, single-pixel FTS was classically known for its ability to obtain very high spectral resolution ( $R$ up to $10^{5}$ ), the "sweet-spot" of the astronomical iFTS clearly sits in the observations of extended emissionline sources at low-to-moderate values of $R$. One can however still exploit the high spectral resolution capability of an iFTS by using narrow-band filters and use it for absorption line studies as well.

The arrival of SITELLE on a $4 \mathrm{~m}$ class telescope (CFHT) under exceptional skies, where it will be used for a wide variety of science projects, will better define the iFTS niche and its capabilities.

\section{Conflict of Interests}

The authors declare that there is no conflict of interests regarding the publication of this paper.

\section{Acknowledgments}

We would like to acknowledge financial contributions from the Canadian Foundation for Innovation, the Canadian Space Agency, the Natural Sciences and Engineering Council of Canada, the Fonds Québécois de la Recherche sur la Nature et les Technologies, the Canada-France-Hawaii Telescope, and Université Laval. We also thank Ghislain Turcotte, Bernard Malenfant, and Pierre-Luc Lévesque for their help at the telescope and the CFHT team led by Marc Baril for their exquisite work on the detector system for SITELLE and its implementation at the telescope. 


\section{References}

[1] J. C. Mather, E. S. Cheng, R. E. Eplee Jr. et al., "A preliminary measurement of the cosmic microwave background spectrum by the cosmic background explorer (COBE) satellite," The Astrophysical Journal, vol. 354, pp. L37-L40, 1990.

[2] H. P. Gush, M. Halpern, and E. H. Wishnow, "Rocket measurement of the cosmic-background-radiation mm-wave spectrum," Physical Review Letters, vol. 65, no. 5, pp. 537-540, 1990.

[3] N. Scoville, D. N. B. Hall, S. G. Kleinmann, and S. F. Ridgway, "Detection of CO band emission in the Becklin-Neugebauer object," The Astrophysical Journal, vol. 232, pp. L121-L124, 1979.

[4] S. T. Ridgway, D. F. Carbon, D. N. B. Hall, and J. Jewell, "An atlas of late-type stellar spectra, 2400-2778 inverse centimeters," The Astrophysical Journal, Supplement Series, vol. 54, pp. 177209, 1984.

[5] A. A. Chalabaev and J. P. Maillard, "Near-infrared spectroscopy of gamma cassiopeiae-constraints on the velocity field in the envelope," The Astrophysical Journal, vol. 294, pp. 640-645, 1985.

[6] J. P. Maillard, J. Crovisier, T. Encrenaz, and M. Combes, "The spectrum of comet p/Halley between 0.9-MICRONS and 2.5MICRONS," Astronomy and Astrophysics, vol. 187, p. 398, 1987.

[7] J. P. Maillard and D. Simons, "Progress in telescope and instrumentation technologies," in Proceedings of the ESO Conference Workshop, p. 733, 1992.

[8] T. Paumard, J.-P. Maillard, and M. Morris, "Kinematic and structural analysis of the minispiral in the galactic center from BEAR spectro-imagery," Astronomy and Astrophysics, vol. 426, no. 1, pp. 81-96, 2004.

[9] R. Wu, E. T. Polehampton, M. Etxaluze et al., "Observing extended sources with the Herschel SPIRE fourier transform spectrometer," Astronomy and Astrophysics, vol. 556, article A116, 11 pages, 2013.

[10] W. Posselt, J.-P. Maillard, and G. Wright, "NIRCAM-IFTS: imaging fourier transform spectrometer for NGST," in NGST Science and Technology Exposition, vol. 207 of Astronomical Society of the Pacific Conference Series, p. 303, 2000.

[11] J. R. Graham, "IFIRS: an imaging Fourier transform spectrometer for the next generation space telescope," in NGST Science and Technology Exposition, vol. 207 of Astronomical Society of the Pacific Conference Series, p. 240, 2000.

[12] S. L. Morris, J. Ouellette, A. Villemaire, F. Grandmont, and L. Moreau, "A Canadian IFTS for the NGST," in NGST Science and Technology Exposition, vol. 207 of Astronomical Society of the Pacific Conference Series, p. 276, 2000.

[13] F. Boulanger, J. P. Maillard, P. Appleton et al., "The molecular hydrogen explorer H2EX," Experimental Astronomy, vol. 23, no. 1, pp. 277-302, 2008.

[14] C. L. Bennett, "Critical comparison of 3-D imaging approaches (review)," in Imaging the Universe in Three Dimensions: Astrophysics with Advanced Multi-Wavelength Imaging Devices, vol. 195 of Astronomical Society of the Pacific Conference Series, p. $58,2000$.

[15] L. Drissen, A. P. Bernier, M. Charlebois, A. Alarie, F. Grandmont, and J. Mandar, "Imaging Fourier transform spectroscopy for astronomy," in Fourier Transforms-New Analytical Approaches and FTIR Strategies, G. Nikolic, Ed., chapter 23, InTech, Rijeka, Croatia, 2011.

[16] J. P. Maillard, L. Drissen, F. Grandmont, and S. Thibault, "Integral wide-field spectroscopy in astronomy: the imaging
FTS solution," Experimental Astronomy, vol. 35, no. 3, pp. 527559, 2013.

[17] T. J. Davidge, "Spectroscopic gradients in bright elliptical galaxies," The Astronomical Journal, vol. 103, no. 5, pp. 1512-1525, 1992.

[18] F. Grandmont, Développement d'un spectromètre imageur à transformée de Fourier pour l'astronomie [Ph.D. thesis], Université Laval, 2007.

[19] D. Lagrois, G. Joncas, and L. Drissen, "Diagnostic line ratios in the IC1805 optical gas complex," Monthly Notices of the Royal Astronomical Society, vol. 420, no. 3, pp. 2280-2299, 2012.

[20] D. Lagrois, L. Drissen, and G. Joncas, Submitted to Monthly Notices of the Royal Astronomical Society.

[21] M. Charlebois, L. Drissen, A.-P. Bernier, F. Grandmont, and L. Binette, "A hyperspectral view of the crab nebula," The Astronomical Journal, vol. 139, no. 5, pp. 2083-2096, 2010.

[22] A. Alarie, A. Bilodeau, and L. Drissen, Monthly Notices of the Royal Astronomical Society. In press.

[23] F. Grandmont, L. Drissen, and G. Joncas, "Development of an imaging Fourier transform spectrometer for astronomy," in Specialized Optical Developments in Astronomy, vol. 4842 of Proceedings of SPIE, pp. 392-401, Waikoloa, Hawaii, USA, August 2002.

[24] A.-P. Bernier, F. Grandmont, J.-F. Rochon, M. Charlebois, and L. Drissen, "First results and current development of SpIOMM: an imaging Fourier transform spectrometer for astronomy," in Ground-Based and Airborne Instrumentation for Astronomy, vol. 6269 of Proceedings of SPIE, p. 135, Orlando, Fla, USA, May 2006.

[25] A.-P. Bernier, L. Drissen, P. Charlebois et al., "Science results from the imaging Fourier transform spectrometer SpIOMM," in 2nd Ground-Based and Airborne Instrumentation for Astronomy, vol. 7014 of Proceedings of SPIE, Marseille, France, June 2008.

[26] L. Drissen, A. Alarie, T. Martin et al., "New scientific results with SpIOMM: a testbed for CFHT's imaging Fourier transform spectrometer SITELLE," in 4th Ground-Based and Airborne Instrumentation for Astronomy, vol. 8446 of Proceedings of SPIE, Amsterdam, The Netherlands, July 2012.

[27] D. A. Thilker, R. Braun, and R. A. M. Walterbos, "HIIphot: automated photometry of $\mathrm{H}$ II regions applied to M51," The Astronomical Journal, vol. 120, no. 6, pp. 3070-3087, 2000.

[28] S. F. Sánchez, F. F. Rosales-Ortega, R. C. Kennicutt et al., "PPAK wide-field integral field spectroscopy of NGC 628-I. The largest spectroscopic mosaic on a single galaxy," Monthly Notices of the Royal Astronomical Society, vol. 410, no. 1, pp. 313340, 2011.

[29] R. C. Kennicutt, J. C. Lee, J. G. Funes, S. Sakai, and S. Akiyama, "An $\mathrm{H} \alpha$ imaging survey of galaxies in the local $11 \mathrm{Mpc}$ volume," The Astrophysical Journal, Supplement Series, vol. 178, no. 2, pp. 247-279, 2008.

[30] C. G. Hoopes, R. A. M. Walterbos, and G. D. Bothun, "Farultraviolet and $\mathrm{H} \alpha$ imaging of nearby spiral galaxies: the $\mathrm{OB}$ stellar population in the diffuse ionized gas," The Astrophysical Journal Letters, vol. 559, no. 2, pp. 878-891, 2001.

[31] R. C. Kennicutt, "Star formation in galaxies along the hubble sequence," Annual Review of Astronomy and Astrophysics, vol. 36, pp. 189-231, 1998.

[32] M. Pettini and B. E. J. Pagel, "[O III]/[N II] as an abundance indicator at high redshift," Monthly Notices of the Royal Astronomical Society, vol. 348, no. 3, pp. L59-L63, 2004. 
[33] L. J. Kewley and M. A. Dopita, "Using strong lines to estimate abundances in extragalactic H II regions and starburst galaxies," The Astrophysical Journal, Supplement Series, vol. 142, no. 1, pp. 35-52, 2002.

[34] G. A. Blanc, A. Heiderman, K. Gebhardt, N. J. Evans II, and J. Adams, "The spatially resolved star formation law from integral field spectroscopy: VIRUS-P observations of NGC 5194," The Astrophysical Journal, vol. 704, no. 1, pp. 842-862, 2009.

[35] L. M. Haffner, R. J. Reynolds, and S. L. Tufte, "WHAM observations of $\mathrm{H} \alpha$, [S II], and [N II] toward the Orion and perseus arms: probing the physical conditions of the warm ionized medium," The Astrophysical Journal, vol. 523, no. 1, pp. 223-233, 1999.

[36] B. Otte, R. J. Reynolds, J. S. Gallagher III, and A. M. N. Ferguson, "Searching for additional heating: [O II] emission in the diffuse ionized gas of NGC 891, NGC 4631, and NGC 3079," The Astrophysical Journal, vol. 560, no. 1, pp. 207-221, 2001.

[37] Y. Liu, X. Zhou, J. Ma, Y. Yang, J. Li, and J. Chen, "Deep surface photometry of M87 with 13 optical bands," The Astrophysical Journal, vol. 129, no. 6, pp. 2628-2635, 2005.

[38] G. Worthey and D. L. Ottaviani, " $\mathrm{H} \gamma$ and $\mathrm{H} \delta$ absorption features in stars and stellar populations," The Astrophysical Journal, Supplement Series, vol. 111, no. 2, pp. 377-386, 1997.

[39] A. Vazdekis, P. Sanchez-Blazquez, J. Falcon-Barroso et al., "Evolutionary stellar population synthesis with MILES-I. The base models and a new line index system," Monthly Notices of the Royal Astronomical Society, vol. 404, no. 4, pp. 1639-1671, 2010.

[40] L. Drissen, A.-P. Bernier, L. Rousseau-Nepton et al., "SITELLE: a wide-field imaging Fourier transform spectrometer for the Canada-France-Hawaii telescope," in 3rd Ground-Based and Airborne Instrumentation for Astronomy, vol. 7735 of Proceedings of SPIE, July 2010.

[41] F. Grandmont, L. Drissen, J. Mandar, S. Thibault, and M. Baril, "Final design of SITELLE: a wide-field imaging Fourier transform spectrometer for the Canada-France-Hawaii telescope," in 4th Ground-Based and Airborne Instrumentation for Astronomy, vol. 8446 of Proceedings of SPIE, October 2012.

[42] T. Martin, L. Drissen, and G. Joncas, "ORBS: a data reduction software for the imaging Fourier transform spectrometers SpIOMM and SITELLE," in 2nd Software and Cyberinfrastructure for Astronomy, vol. 8451 of Proceedings of the SPIE, Amsterdam, The Netherlands, July 2012.

[43] J. R. Graham, M. Abrams, C. Bennett et al., "The performance and scientific rationale for an infrared imaging Fourier transform spectrograph on a large space telescope," Publications of the Astronomical Society of the Pacific, vol. 110, pp. 1205-1215, 1998.

[44] R. Bacon, Y. Copin, G. Monnet et al., "The SAURON project-I. The panoramic integral-field spectrograph," Monthly Notices of the Royal Astronomical Society, vol. 326, no. 1, pp. 23-35, 2001.

[45] H. Kuntschner, E. Emsellem, R. Bacon et al., "The SAURON project-XVII. Stellar population analysis of the absorption line strength maps of 48 early-type galaxies," Monthly Notices of the Royal Astronomical Society, vol. 408, no. 1, pp. 97-132, 2010.

[46] E. Marmol-Queralto, S. F. Sánchez, R. A. Marino et al., "Integral field spectroscopy of a sample of nearby galaxies I. Sample, observations, and data reduction," Astronomy and Astrophysics, vol. 534, article A8, 2011.

[47] S. Sanchez, F. F. Rosales-Ortega, R. A. Marino et al., "Integral field spectroscopy of a sample of nearby galaxies II. Properties of the H II regions," Astronomy and Astrophysics, vol. 546, article A2, 28 pages, 2012 .

[48] J. Iglesias-Paramo, J. M. Vílchez, L. Galbany et al., "Aperture corrections for disk galaxy properties derived from the CALIFA survey: balmer emission lines in spiral galaxies," Astronomy and Astrophysics, vol. 553, article L7, 5 pages, 2013.

[49] G. A. Blanc, A. Heiderman, K. Gebhardt, N. J. Evans II, and J. Adams, "The spatially resolved star formation law from integral field spectroscopy: VIRUS-P observations of NGC 5194," The Astrophysical Journal, vol. 704, no. 1, p. 842, 2009.

[50] G. A. Blanc, T. Weinzirl, M. Song et al., "The VIRUS-P exploration of nearby galaxies (VENGA): survey design, data processing, and spectral analysis methods," The Astrophysical Journal, vol. 145, no. 5, p. 138, 2013.

[51] F. Bresolin, "Clues on the rejuvenation of the S0 galaxy NGC 404 from the chemical abundance of its outer disk," The Astrophysical Journal, vol. 772, no. 2, p. L23, 2013.

[52] L. Kewley and H. J. Zahid, "Strong-line methods for oxygen abundance determinations in giant HII regions and emission line galaxies: methods and uncertainties," Mapping Oxygen in the Universe, 2012, http://www.iac.es/congreso/ oxygenmap/pages/electronic-presentations.php. 

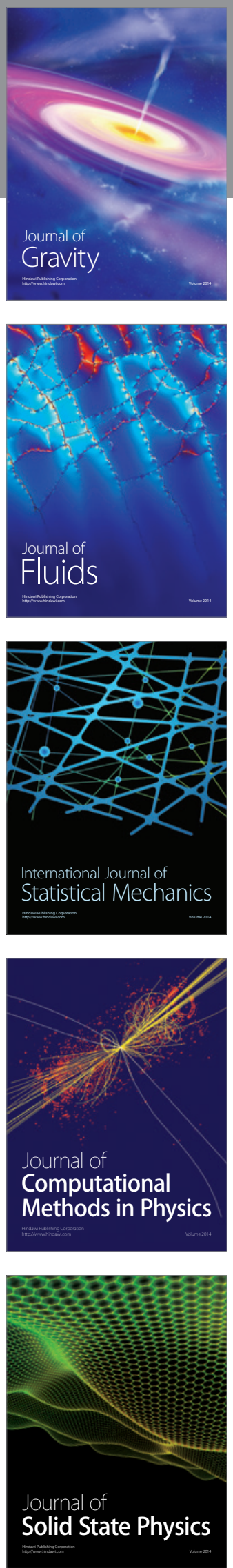

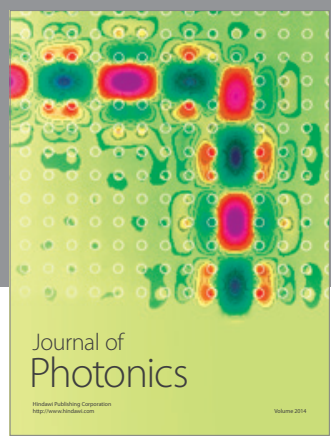

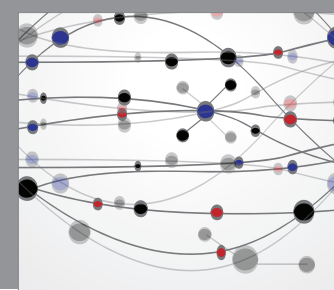

The Scientific World Journal

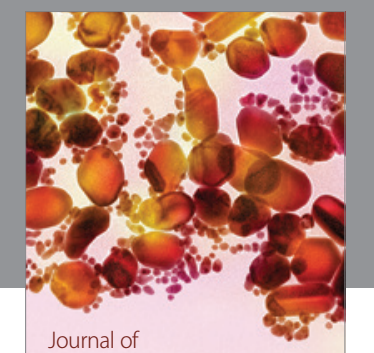

Soft Matter
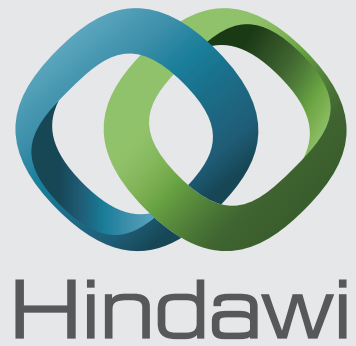

Submit your manuscripts at

http://www.hindawi.com
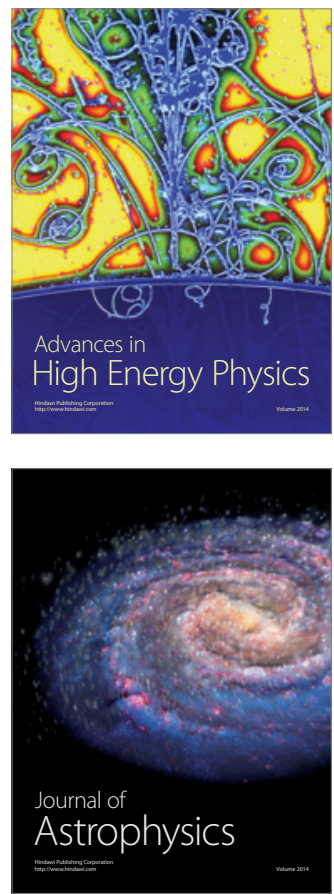
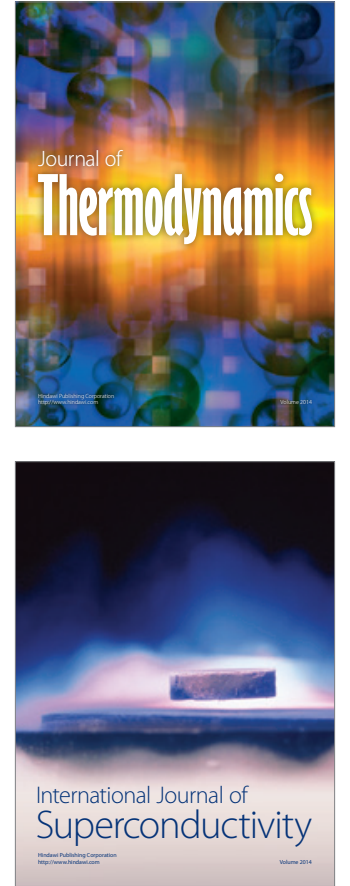
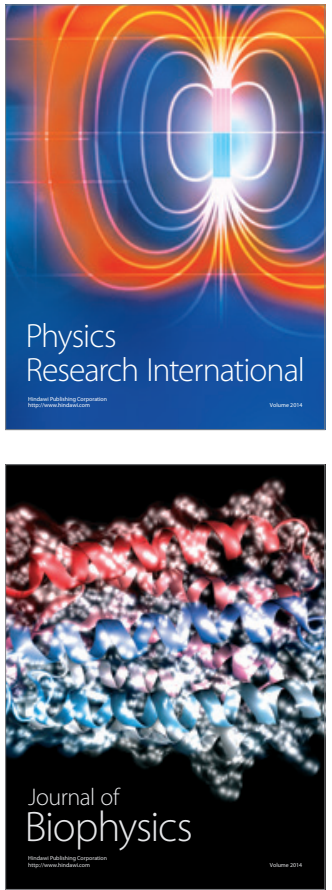
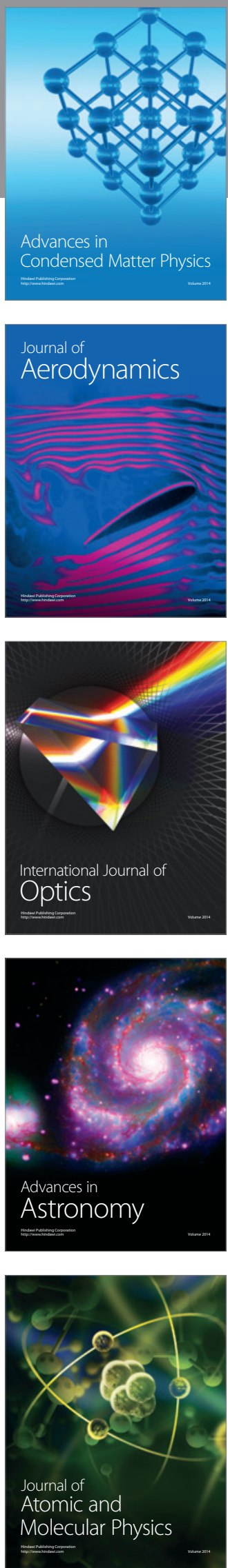